\title{
Finite-time Blow-up of Solutions of Some Long-wave Unstable Thin Film Equations
}

\author{
A. L. BerTozZI AND M. C. PUGH
}

ABSTRACT. We consider the family of long-wave unstable lubrication equations

$$
h_{t}=-\left(h h_{x x x}\right)_{x}-\left(h^{m} h_{x}\right)_{x}
$$

with $m \geq 3$. Given a fixed $m \geq 3$, we prove the existence of a weak solution that becomes singular in finite time. Specifically, given compactly supported nonnegative initial data with negative energy, there is a time $T^{*}<\infty$, determined by $m$ and the $H^{1}$ norm of the initial data, and a compactly supported nonnegative weak solution such that $\lim \sup _{t \rightarrow T^{*}}\|h(\cdot, t)\|_{L^{\infty}}=$ $\lim \sup _{t \rightarrow T^{*}}\|h(\cdot, t)\|_{H^{1}}=\infty$. We discuss the relevance of these singular solutions to an earlier conjecture [Comm. Pure. Appl. Math. 51 (1998), 625-661] on when finite-time singularities are possible for long-wave unstable lubrication equations.

\section{INTRODUCTION}

The study of finite-time singularities in nonlinear PDEs is important in problems ranging from inviscid incompressible fluid flow $[15,30]$ to concentrations in bacterial colonies [14]. A common theme is the role of scaling and self-similarity in determining the existence and structure of singularities. A classical example that shows the role of scaling is the semilinear heat equation

$$
h_{t}=\Delta h+h^{p}
$$

and its degenerate generalization

$$
h_{t}=\Delta\left(h^{m}\right)+h^{p} .
$$


A comprehensive discussion of how scaling properties of the equations relate to singularity formation can be found in [39]. Both equations possess a maximum principle which helps in proving strong results such as the universality of selfsimilar blow-up for the initial value problem.

In contrast, fourth-order analogues of these equations do not possess a maximum principle. An important class of examples is the Cahn-Hilliard equation and its brethren; here, like in the above equations, a long-wave instability can couple with the nonlinearity to yield finite-time blow-up. Hocherman and Rosenau [25] considered a family that included equations of the form:

$$
h_{t}=-\left(f(h) h_{x x x}\right)_{x}-\left(g(h) h_{x}\right)_{x} .
$$

This equation is long-wave unstable if $f$ and $g$ are nonnegative: Perturbing around a positive constant steady state, the fourth-order term is linearly stabilizing and the second-order term is linearly destabilizing. They were interested in when such equations admit solutions that blow up in finite time: $\lim \sup _{t \rightarrow T^{*}}\|h(\cdot, t)\|_{\infty}=$ $\infty$. They conjectured that the large- $y$ behavior of $g(y) / f(y)$ determines the presence or absence of a finite-time blow-up:

$$
\lim _{y \rightarrow \infty} \frac{g(y)}{f(y)}= \begin{cases}\infty: & h \rightarrow \infty \text { in finite time for some solutions } \\ \text { finite }: & \text { marginal case } \\ 0: & \text { globally stable solutions. }\end{cases}
$$

Their conjecture addressed a larger class of equations including those of form (1). Also, their conjecture included both degenerate coefficients $(f(y) \rightarrow 0$ as $y \rightarrow 0)$ and nondegenerate coefficients $(f(y) \rightarrow f(0)>0$ as $y \rightarrow 0)$.

In a recent paper [13], we considered the possible formation of finite-time singularities in the subclass of degenerate problems. Such degeneracy can lead to a nonnegativity principle: Initially nonnegative solutions remain nonnegative. Also, since equation (1) is in divergence form, the evolution conserves the area of the solution. The following scaling argument takes into account both this volume conservation and the nonnegativity of solutions, suggesting a modified conjecture for the degenerate case.

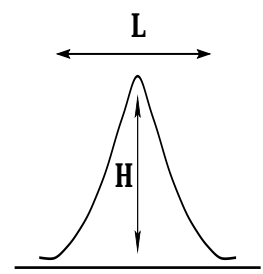

Consider a solution with a height-scale $H$ and length-scale $L$. Nonnegativity and volume conservation require that $H L \leq V$, where $V$ is the total fluid volume. 
The critical regime for the evolution equation should correspond to a balance between the nonlinear terms in equation (1)

$$
\frac{f(H) H}{L^{4}} \sim \frac{g(H) H}{L^{2}} \Rightarrow \frac{f(H)}{g(H)} \sim L^{2} .
$$

From the volume constraint, this implies $H^{2} f(H) / g(H)<V^{2}$. This suggests that the solution can grow without bound only if $\lim _{y \rightarrow \infty} y^{2} f(y) / g(y)<\infty$. Including the $h_{t}$ term of equation (1) in the scaling argument yields

$$
\dot{H} \leq \frac{g(H) H}{L^{2}} \sim \frac{g(H)^{2}}{f(H)} H .
$$

This suggests that any blow-up must take infinite time whenever $\lim _{y \rightarrow \infty} g(y)^{2} / f(y)=A<\infty$ since the solution would then be dominated by $e^{A t}$. These scaling arguments led to the following result:

Conjecture. ([13]) Consider the evolution equation

$$
h_{t}=-\left(f(h) h_{x x x}\right)_{x}-\left(g(h) h_{x}\right)_{x} .
$$

Assume $f$ and $g$ are nonnegative, $g(y) / f(y)$ is bounded as $y \downarrow 0$, and $f$ is degenerate: $f(y) \rightarrow 0$ as $y \downarrow 0$. Then the large-y behavior of $g(y) /\left(y^{2} f(y)\right)$ determines the presence or absence of blow-up for nonnegative solutions:

$$
\lim _{y \rightarrow \infty} \frac{g(y)}{y^{2} f(y)}= \begin{cases}\infty: & \text { supercritical: blow-up possible } \\ \text { finite : } & \text { critical case } \\ 0: & \text { subcritical: solutions are globally bounded. }\end{cases}
$$

If $\lim _{y \rightarrow \infty} g(y) / \sqrt{f(y)}=\infty$, then it is possible that the blow-up will occur in finite time.

For power-law coefficients $f(y)=y^{n}$ and $g(y)=y^{m}$, the conjecture of Hocherman and Rosenau suggests that if $m>n$, then finite-time blow-up is possible. Our conjecture suggests that if $0<n \leq m<n+2$, then nonnegative solutions are bounded for all time. We proved the uniform boundedness part of the conjecture for positive smooth solutions for all $n$ and for nonnegative weak solutions when $0<n<3$ [13]. ${ }^{1}$ For the supercritical case $(m>n+2)$, we provided numerical evidence suggesting finite-time blow-up was possible and that the conjecture is sharp [13]. The condition $m \geq n$ is a 'well-posedness' condition

\footnotetext{
${ }^{1}$ Their conjecture certainly still stands for equations with nondegenerate fourth-order terms: In fact, finite-time singularities have been proven to exist for some equations with $m=n+1[7,13]$.
} 
that ensures the fourth order diffusion dominates the second order 'anti-diffusion' in the limit as $h \rightarrow 0$. Heuristically we expect this condition to be necessary for well-posed dynamics, otherwise the problem behaves like a backward degenerate diffusion equation near the contact line. Further, we find that the estimates needed for our existence methods seem to require that the condition $m \geq n$ hold.

In [13] we state the conjecture for periodic solutions. It is natural to extend this conjecture (unchanged) to the problem on the line since compactly supported initial data yield solutions whose support has finite speed of propagation (Theorem 2). Indeed, we use this equivalence between the periodic problem and the problem on the line to prove existence of compactly supported solutions to the Cauchy problem. We proved the subcritical part of the conjecture for periodic nonnegative weak solutions for a subclass of subcritical coefficients $f$ and $g$. This includes the case $f(y)=y$ and $g(y)=y^{m}$ with $m<3$.

Here, we consider solutions on the line and prove that our conjecture is sharp for nonnegative solutions of the equation

$$
h_{t}=-\left(h h_{x x x}\right)_{x}-\left(h^{m} h_{x}\right)_{x} .
$$

Proving the sharpness for solutions on the line involves two steps. First, we prove that for the subcritical case, uniformly bounded compactly supported nonnegative weak solutions exist on the line for all time (Proposition 3). Second, we prove that given any $m \geq 3$, there exists a nonnegative compactly supported weak solution on the line that becomes singular in finite time:

Theorem. Let $h_{0}$ be nonnegative and compactly supported, $h_{0} \in H^{1}(\mathbb{R})$. If $m \geq 3$ and

$$
\mathcal{E}(0)=\frac{1}{2} \int_{-\infty}^{\infty} h_{0 x}^{2}(x) d x-\frac{1}{m(m+1)} \int_{-\infty}^{\infty} h_{0}^{m+1}(x) d x<0,
$$

then there is a singular time $T^{*}<\infty$ and a compactly supported nonnegative weak solution in the sense of distributions (5) on $\left[0, T^{*}\right)$ such that

$$
\limsup _{t \rightarrow T^{*}}\|h(\cdot, t)\|_{L^{\infty}(\mathbb{R})}=\limsup _{t \rightarrow T^{*}}\|h(\cdot, t)\|_{H^{1}(\mathbb{R})}=\infty .
$$

We prove finite-time blow-up for both the critical $m=3$ case and for the supercritical $m>3$ case. As we discuss in the conclusions, Section 5, the two cases have self-similar solutions with markedly different properties, and numerical simulations of the initial value problem suggest solutions blow up in distinct manners.

An outline of the proof is as follows:

- Given $m \geq 3$ and nonnegative compactly supported initial data $h_{0}$ supported in $(-a, a)$, then $m, a$, and $\left\|h_{0}\right\|_{H^{1}}$ determine a time of existence, $T_{0}>0$. There exists a periodic nonnegative weak solution $h$ on $[-a, a] \times$ $\left[0, T_{0}\right]$ (Theorem 1). 
- The time of existence $T_{0}$ can be taken to depend on $m$ and $\left\|h_{0}\right\|_{H^{1}}$ only (equation (40)).

- Given compactly supported initial data, the above solution has finite speed of propagation of the support. This speed is bounded by a function of $\left\|h_{0}\right\|_{H^{1}}$ and $m$. By taking the interval $[-a, a]$ sufficiently large, one can extend the periodic weak solution to a weak solution on $\mathbb{R} \times\left[0, T_{0}\right]$ (Proposition 2).

- This solution can be continued in time if either the $L^{\infty}$ or the $H^{1}$ norm of $h$ at time $T_{0}$ is finite (Theorem 2). The $L^{\infty}$ norm is continuous in time.

- There is some time $T^{*}$, determined by $m$ and $\left\|h_{0}\right\|_{H^{1}}$, past which this solution cannot exist. It then follows that the $H^{1}$ and $L^{\infty}$ norm of the solution must have blown up at or before the time $T^{*}$ (Theorem 3 ).

The nonexistence time $T^{*}$ follows from a second-moment argument, found formally by Andrew Bernoff [6]. One of our results is that compactly supported nonnegative weak solutions satisfy the following second-moment inequality:

Lemma. There is a sequence of times $0<T_{0}<\cdots<T_{i}<\cdots<\infty$ such that the compactly supported nonnegative weak solution satisfies the second-moment inequality

$$
\begin{aligned}
\int_{-\infty}^{\infty} x^{2} h\left(x, T_{i}\right) d x \leq & \int_{-\infty}^{\infty} x^{2} h_{0}(x) d x+6 \mathcal{E}(0) T_{i} \\
& -\frac{2(m-3)}{m(m+1)} \int_{0}^{T_{i}} \int_{-\infty}^{\infty} h^{m+1}(x, t) d x d t
\end{aligned}
$$

where

$$
\mathcal{E}(t)=\frac{1}{2} \int_{-\infty}^{\infty} h_{x}^{2}(x, t) d x-\frac{1}{m(m+1)} \int_{-\infty}^{\infty} h^{m+1}(x, t) d x
$$

It follows immediately that if $m \geq 3$, then at the times $T_{i}$

$$
\int_{-\infty}^{\infty} x^{2} h\left(x, T_{i}\right) d x \leq \int_{-\infty}^{\infty} x^{2} h_{0}(x) d x+6 \mathcal{E}(0) T_{i} .
$$

Given initial data $h_{0}$ for which $\mathcal{E}(0)<0$, if $T_{i} \rightarrow \infty$ then the right-hand side would become negative, which is impossible. Therefore $T_{i} \rightarrow T^{*}<\infty$. In the construction, the times $T_{i}$ are the end-times of intervals of existence: Specifically, $T_{i+1}-T_{i}$ is an explicit function of the exponent $m$ and the $H^{1}$ norm of the solution at time $T_{i}$. We prove their having a finite limit implies the constructed solution must blow up. While the blow-up argument is straightforward, much of the effort in this article involves proving that the nonnegative compactly supported weak solution satisfies the above second-moment inequality. Also, we note that the blow-up result is somewhat striking in that although we do not prove the $H^{1}$ norm 
of the solution is continuous in time, we prove that it is blowing up at a sequence of times. The $L^{\infty}$ norm is continuous in time and is also blowing up.

This (constructed) solution lives in a particular regularity class of "strong" weak solutions (see Section 3). To date, there is no proof of uniqueness of weak solutions in the regularity class determined by Theorem 2 . For this reason, while we believe this solution cannot be continued as a weak solution past the time $T^{*}$, we cannot exclude the possibility that the same initial data might yield a different solution that exists beyond the time $T^{*}$. Numerical studies of the initial value problem robustly show finite-time blow-up of the following form: It is focused at a single point away from the edge of the support. This describes the large-scale structure of the blow-up for both the critical and super-critical cases; however their fine structure, such as scaling properties, differ [8].

Using functionals of the solution to prove finite-time blow-up of solutions was first introduced by Levine [29] in his study of nonlinear wave equations. As he wrote there, a key aspect was having a system where the energy was unsigned and contained two terms with opposite sign, one corresponding to potential energy and the other to kinetic energy. Glassey [21] used a functional involving the variance of the solution, $\int x^{2}|h(x, t)|^{2} d x$, to prove blow-up for the nonlinear Schrödinger equation.

\section{BACKGROUND AND PRIOR WORK}

Among other places, degenerate fourth-order equations arise in the study of thin liquid films driven by surface tension. The simplest example is a thin viscous layer of liquid on a flat surface. The air/liquid interface is at $z=h(x, y, t)$ and the liquid/solid interface at $z=0$. At each point on the air/liquid interface, the pressure jump is proportional to the curvature of the interface at that point. If one assumes the flow is uniform in the $y$-direction, the evolution of the film thickness can be modeled by

$$
h_{t}=-\left(f(h) h_{x x x}\right)_{x},
$$

where $f(h)=h^{3}+\beta h^{n}$ and $n>0[18,23,24,33]$. We refer readers interested in the physical and mathematical aspects of this and similar equations to review articles $[9,26,31,33]$.

Since $h$ represents the thickness of the liquid film, it should be a nonnegative quantity. This makes it important to prove that nonnegative initial data yield nonnegative solutions; the degeneracy, $f(h) \rightarrow 0$ as $y \rightarrow 0$, is key in proving this.

A well-known second-order degenerate parabolic equation is the porous medium equation

$$
h_{t}=(\Phi(h))_{x x},
$$

where $\Phi^{\prime}(h)>0$ for $h>0$, and $\Phi(h) \sim h^{m}$ as $h \rightarrow 0$ with $m>1$. This equation has been studied by a many researchers; we refer the reader to [38] for references. It enjoys the following properties: 
- instantaneous smoothing of the solution in regions of positive $h$,

- a maximum principle,

- finite speed of propagation of the support of the solution,

- well-posed weak solutions for smooth nonnegative initial data.

Equation (3), a fourth-order analogue of the porous medium equation, satisfies some related properties. We address each property in turn.

First of all, the evolution equation is uniformly parabolic where the solution is bounded away from zero [5]. As a result, the solution is $C^{\infty}$-smooth at points where it is strictly positive, but can be less regular at the boundary of its support. Also, if a strictly positive solution becomes zero at some point in finite time, there must be an accompanying loss of regularity. Indeed, as discussed in [17], given any equation in flux form $h_{t}+(h U)_{x}=0$, if a smooth positive solution becomes zero at a point in finite time $T^{*}$, then

$$
\int_{0}^{T^{*}} U_{x}\left(x_{\min }(t), t\right) d t=\infty
$$

where $x_{\min }(t)$ is the position at time $t$ of the local minimum that is touching down. For $h_{t}=-\left(h h_{x x x}\right)_{x}$ this means that at the very least $h_{x x x x}$ would blow up in finite time. For equation (3) the positive classical solution can then be continued in time as a nonnegative weak solution $[1,5,12]$.

The maximum principle is not satisfied by fourth-order diffusion equations. For $h_{t}=-h_{x x x x}$, positive initial data can lead to solutions that change sign. However, if equation (3) is sufficiently degenerate, then there is a nonnegativity principle. Specifically, for $f(h)=h^{n}$ with $n \geq 3.5$, if the initial condition $h_{0}$ is positive, then a periodic solution $h(x, t)$ will stay positive [10, for $n \geq 3.5$ ], [5, for $n \geq 4]$. (Numerical simulations of periodic solutions suggest there is a critical exponent $n_{c} \in(1,2)$ such that if $n>n_{c}$, then initially positive solutions remain positive [10].) Also, nonnegative initial data yield nonnegative "strong" weak solutions of equation (3) for $0<n<3[1,5,12]$. The nonnegativity principle is not solely due to the degeneracy; other fourth-order degenerate diffusion equations have weak solutions that change sign [2].

Another physical property is finite speed of propagation. Compactly supported initial data should yield a solution whose contact line (where air, liquid, and solid meet) moves with finite speed. Bernis proved this for $0<n<3$ in $[3,4]$.

Finally, concerning well-posedness, there are simple counter-examples [1] to uniqueness of solutions. These counter-examples involve solutions like the steady state $h(x)=[(a-x)(x-b)]_{+}$that are not $C^{1}$ at the contact line. It may be that solutions are unique within the class of 'strong solutions': solutions that are not classical but are continuous in time and are $C^{1}$ at the contact line at almost all times. The weak solution we construct here is such a strong solution. 
In general, the proofs of the above properties rely heavily on energy methods. The exception is Otto's work on $h_{t}=-\left(h h_{x x x}\right)_{x}$ in which he uses variational methods [35].

Equation (3) with periodic or zero-flux boundary conditions has very simple long-time dynamics: Solutions spread and flatten until they become completely flat $[1,12]$. More complex dynamics occur when destabilizing forces are present. This often enters as a destabilizing second-order term, resulting in equation (1). Unlike equation (3), whose only periodic steady state is constant, the long-wave unstable equation (1) can have a variety of nontrivial steady states [28, 32]. Given a fixed period and volume, there can be both linearly stable and linearly unstable steady states with that period and volume [27]. Also, as we prove here, in addition to ever-possible $h \rightarrow 0$ singularities, the long-wave unstable equation (1) can have $h \rightarrow \infty$ in finite time: We believe it is not possible to continue the weak solution past this time.

\section{SHORT-TIME EXISTENCE OF COMPACTLY-SUPPORTED WEAK SOLUTIONS ON THE LINE}

In this section we consider the initial value problem for

$$
h_{t}=-\left(h h_{x x x}\right)_{x}-\left(h^{m} h_{x}\right)_{x}
$$

with compactly supported initial data $h_{0} \in H^{1}(\mathbb{R})$. We prove that there exists a time $T_{0}$, depending only on $m$ and the $H^{1}$ norm of the initial data, such that the initial data $h_{0}$ yields a weak solution $h$ of (4) in the following sense of distributions:

$$
\begin{gathered}
-\int_{0}^{T_{0}} \int_{-\infty}^{\infty} h(x, t) \varphi_{t}(x, t) d x d t+\int_{-\infty}^{\infty} h\left(x, T_{0}\right) \varphi\left(x, T_{0}\right) d x \\
-\int_{-\infty}^{\infty} h_{0}(x) \varphi(x, 0) d x \\
=\int_{0}^{T_{0}} \int_{-\infty}^{\infty} \varphi_{x x x}(x, t) h(x, t) h_{x}(x, t) d x d t \\
-\frac{1}{m+1} \int_{0}^{T_{0}} \int_{-\infty}^{\infty} \varphi_{x x}(x, t) h^{m+1}(x, t) d x d t \\
+\frac{3}{2} \int_{0}^{T_{0}} \int_{-\infty}^{\infty} \varphi_{x x}(x, t) h_{x}^{2}(x, t) d x d t
\end{gathered}
$$

for all compactly supported test functions: $\varphi$ in $C_{\mathcal{C}}^{\infty}\left(\mathbb{R} \times\left[0, T_{0}\right]\right)$. The weak solution $h$ is in

$$
L^{2}\left(0, T_{0} ; H^{2}(\mathbb{R})\right) \cap L^{\infty}\left(0, T_{0} ; H^{1}(\mathbb{R})\right) .
$$

This weak form of the equation is different from those considered in $[5,12,13]$ in that only $h$ and its first spatial derivatives are expressed explicitly in the weak 
form. It bears closest resemblance to the weak forms considered in $[12,13]$. The weak form (5) makes explicit use of the fact that $f(h)=h$ (in (1)) and therefore $f^{\prime \prime}=0$. Also, the test functions are not required to be zero for times near $t=0$; $\varphi(x, t)$ can be nonzero at both $t=0$ and $t=T$. These differences are crucial for obtaining the second moment inequality used to prove finite-time blow-up.

We prove existence on the line by first proving existence of a periodic nonnegative weak solution on an interval $[-a, a]$ containing the support of the initial data (see Section 3.1). The periodic solution exists up to a time $T_{0}$ determined by $m$, the interval length $a$, and $\left\|h_{0}\right\|_{H^{1}}$. We then prove that the support of the solution has finite speed of propagation, $V$ (see Section 3.3). The speed $V$ is bounded above by a function of $m$ and the $H^{1}$ norm of the initial data. This allows us to choose the interval $[-a, a]$ sufficiently large so that at the time $T_{0}$, the support of the solution remains in a compact subset of $(-a, a)$. Hence the periodic solution can be extended by zero from $(-a, a)$ to $\mathbb{R}$ (see Section 3.4).

Notation. Throughout this section, $\|\cdot\|_{H^{1}}$ refers the the norm in $H^{1}(-a, a)$.

3.1. Short-time existence of periodic nonnegative weak solutions. We prove the existence of a nonnegative periodic weak solution by approximating it with smooth solutions of an approximate equation. For the porous medium equation, $h_{t}=\left(h^{m} h_{x}\right)_{x}, m>0$, a natural approximate equation is the uniformly parabolic equation $h_{\varepsilon t}=\left(\left(h_{\varepsilon}{ }^{m}+\varepsilon\right) h_{\varepsilon x}\right)_{x}$. This equation is second-order, and the maximum principle implies positive initial data yield positive solutions. A subsequence of these positive approximate solutions will have a nonnegative $\varepsilon \rightarrow 0$ limit, which is then proved to be a weak solution of the original equation. The equation $h_{t}=-\left(h^{n} h_{x x x}\right)_{x}$ is a fourth-order equation. Because the maximum principle does not apply to strictly parabolic fourth-order equations, the approximate equation $h_{\varepsilon t}=-\left(\left(h_{\varepsilon}{ }^{n}+\varepsilon\right) h_{\varepsilon x x x}\right)_{x}$ can take positive initial data to solutions that are negative in regions. It is not obvious that the limit of such solutions would be nonnegative. For this reason and for reasons concerning the numerical approximation of solutions, we use a degenerate approximate equation, one that has been chosen so that positive initial data yield positive solutions. These approximation issues are also relevant for the long-wave unstable equation (1) we consider here. The regularization we use is a modification of that introduced in Bernis and Friedman for the purely fourth-order equation [5].

3.2. The approximate problem. We use the approximate problem:

$$
\begin{gathered}
h_{t}=-\left(f_{\varepsilon}\left(h_{\varepsilon}\right) h_{\varepsilon x x x}\right)_{x}-\left(g_{\varepsilon}\left(h_{\varepsilon}\right) h_{\varepsilon x}\right)_{x}, \\
h_{\varepsilon}(x, 0)=h_{\varepsilon 0}(x)=h_{0}(x)+\delta(\varepsilon)>0, \\
h(\cdot, t) \text { periodic on }[-a, a],
\end{gathered}
$$

where $\delta(\varepsilon)=\varepsilon^{9} a^{-1 / 2}, \vartheta<\frac{2}{5}$, and $0<\varepsilon<1$. The constraint on 9 is used to guarantee a 'zero contact angle' weak solution in the limit as $\varepsilon \rightarrow 0$ (see e.g. 
$[1,12])$. The initial data has been "lifted" so that $h_{\varepsilon 0}$ is strictly positive. As we discuss shortly, the dependence of $\delta(\varepsilon)$ on the interval size $a$ has been chosen so that the a priori bounds can be taken independent of $\varepsilon$. The coefficients $f_{\varepsilon}$ and $g_{\varepsilon}$ are

$$
f_{\varepsilon}(y)=\frac{y^{5}}{\varepsilon y+y^{4}} \quad \text { and } \quad g_{\varepsilon}(y)= \begin{cases}\frac{y^{m+4}}{\varepsilon y^{m}+y^{4}}, & m<4, \\ y^{m}, & m \geq 4 .\end{cases}
$$

The regularization $f_{\varepsilon}$ was introduced in [5] and later used in [1, 12], for equation (3). As in [13], the second-order term $-\left(h^{m} h_{x}\right)_{x}$ must also be regularized because it enters the equation in a linearly destabilizing manner. Both regularizations leave the large- $y$ asymptotics unchanged, $f_{\varepsilon}(y) \sim y, g_{\varepsilon}(y) \sim y^{m}$, while the small- $y$ asymptotics are $f_{\varepsilon}(y) \sim y^{4}$, and $g_{\varepsilon}(y)$ is at least as degenerate as $f_{\varepsilon}(y)$. This is the same regularization that is used to prove the existence of nonnegative periodic weak solutions for the subcritical case $(m<n+2)$ of our conjecture [13].

We first prove a priori bounds for smooth periodic solutions of the approximate problem:

Lemma 1. (A priori $H^{1}$ bound of approximate solutions) Let $h_{\varepsilon}$ be a smooth periodic solution of the approximate problem (6) on $[-a, a]$ with $m \geq 3$. Then there is a constant $C_{a}$ that depends on $m$ and $a$ such that, on the interval $\left[0, T_{m \varepsilon}\right)$ with

$$
T_{m \varepsilon}:=\frac{1}{C_{a}(2 m-1)} \min \left\{1,\left\|h_{\varepsilon 0}\right\|_{H^{1}}^{1-2 m}\right\},
$$

the solution satisfies the bound

$$
\left\|h_{\varepsilon}(\cdot, t)\right\|_{H^{1}}^{2} \leq y(t)<\infty,
$$

where $d y / d t=C_{a}\left(y^{3 / 2}+y^{(2 m+1) / 2}\right)$ and $y(0)=\left\|h_{\varepsilon 0}\right\|_{H^{1}}^{2}$. The solution also satisfies the bound

$$
\left\|h_{\varepsilon}(\cdot, t)\right\|_{H^{1}}^{2} \leq\left\|h_{\varepsilon 0}\right\|_{H^{1}}^{2} e^{A(t)},
$$

where

$$
A(t):=C \int_{0}^{t}\left\|h_{\varepsilon}(\cdot, \tau)\right\|_{L^{\infty}}+\left\|h_{\varepsilon}(\cdot, \tau)\right\|_{L^{\infty}}^{2 m-1} d \tau .
$$

The a-dependence of $C_{a}$ is $C_{a}=C\left(c_{\infty}+1 / a\right)^{2 m-1}$.

Remark. The proof of Lemma 1 holds for $m>1$. However, for $1<m<3$ there is a uniform upper bound on $\left\|h_{\varepsilon}(\cdot, t)\right\|_{H^{1}}$ [13]. For this reason, we state the lemma and its consequences only for $m \geq 3$. Also, we note that $C_{a}$ remains bounded as $a \rightarrow \infty$. 
Proof. The $H^{1}$ norm satisfies

$$
\begin{aligned}
& \frac{d}{d t}\left(\frac{1}{2} \int_{-a}^{a} h_{\varepsilon x}^{2}(x, t) d x+\frac{1}{2} \int_{-a}^{a} h_{\varepsilon}^{2}(x, t) d x\right) \\
& =-\int_{-a}^{a} f_{\varepsilon}\left(h_{\varepsilon}(x, t)\right)\left(h_{\varepsilon x x x}(x, t)\right. \\
& \left.\quad-\frac{f_{\varepsilon}\left(h_{\varepsilon}(x, t)\right)-g_{\varepsilon}\left(h_{\varepsilon}(x, t)\right)}{2 f_{\varepsilon}\left(h_{\varepsilon}(x, t)\right)} h_{\varepsilon x}(x, t)\right)^{2} d x \\
& \quad+\int_{-a}^{a} \frac{\left(f_{\varepsilon}\left(h_{\varepsilon}(x, t)\right)+g_{\varepsilon}\left(h_{\varepsilon}(x, t)\right)\right)^{2}}{4 f_{\varepsilon}\left(h_{\varepsilon}(x, t)\right)} h_{\varepsilon x}^{2}(x, t) d x \\
& \leq\left\|\frac{\left(f_{\varepsilon}\left(h_{\varepsilon}(\cdot, t)\right)+g_{\varepsilon}\left(h_{\varepsilon}(\cdot, t)\right)\right)^{2}}{4 f_{\varepsilon}\left(h_{\varepsilon}(\cdot, t)\right)}\right\|_{L^{\infty}} \int_{-a}^{a} h_{\varepsilon x}^{2}(x, t) d x \\
& \leq C\left(\left\|h_{\varepsilon}(\cdot, t)\right\|_{L^{\infty}}+\left\|h_{\varepsilon}(\cdot, t)\right\|_{L^{\infty}}^{2 m-1}\right)\left\|h_{\varepsilon}\right\|_{H^{1}}^{2} \\
& \leq C\left(c_{a}\left\|h_{\varepsilon}(\cdot, t)\right\|_{H^{1}}^{3}+c_{a}^{2 m-1}\left\|h_{\varepsilon}(\cdot, t)\right\|_{H^{1}}^{2 m+1}\right) \\
& \leq C c_{a}^{2 m-1}\left(\left\|h_{\varepsilon}(\cdot, t)\right\|_{H^{1}}^{3}+\left\|h_{\varepsilon}(\cdot, t)\right\|_{H^{1}}^{2 m+1}\right) .
\end{aligned}
$$

At step (9), we use the fact that

$$
\frac{\left(f_{\varepsilon}(y)+g_{\varepsilon}(y)\right)^{2}}{4 f_{\varepsilon}(y)} \sim \frac{1}{4} y^{2 m-1} \quad \text { for } y \gg 1
$$

and

$$
\frac{\left(f_{\varepsilon}(y)+g_{\varepsilon}(y)\right)^{2}}{4 f_{\varepsilon}(y)} \rightarrow \frac{1}{4} y+\frac{1}{2} y^{m}+\frac{1}{4} y^{2 m-1} \quad \text { for } y \in[0,1]
$$

uniformly in $\varepsilon$. It then follows that there is some constant independent of $\varepsilon$ such that

$$
\frac{\left(f_{\varepsilon}(y)+g_{\varepsilon}(y)\right)^{2}}{4 f_{\varepsilon}(y)} \leq C\left(y+y^{2 m-1}\right) .
$$

At step (10), we bounded the $L^{\infty}$ norm with the $H^{1}$ norm:

$$
\|h\|_{L^{\infty}([-a, a])} \leq c_{a}\|h\|_{H^{1}([-a, a])}=\left(c_{\infty}+\frac{1}{a}\right)\|h\|_{H^{1}([-a, a])} .
$$

We take $c_{\infty} \geq 1$ so that $c_{a}<c_{a}^{2 m-1}$ in step (10).

From (10), $\left\|h_{\varepsilon}(\cdot, t)\right\|_{H^{1}}^{2}$ is dominated by the solution of

$$
\begin{aligned}
\frac{d y}{d t} & =C_{a}\left(y(t)^{3 / 2}+y(t)^{(2 m+1) / 2}\right), \\
y(0) & =\left\|h_{\varepsilon 0}\right\|_{H^{1}}^{2},
\end{aligned}
$$


where $C_{a}=C c_{a}^{2 m-1}$. If $y(0)<1$, then there is some time $t_{0}>0$ at which $y\left(t_{0}\right)=1$. If $y(0) \geq 1$, then take $t_{0}=0$. For $t \geq t_{0}, y(t)$ is dominated by $z(t)$, the solution of

$$
\begin{aligned}
\frac{d z}{d t} & =2 C_{a} z(t)^{(2 m+1) / 2}, \\
z\left(t_{0}\right) & =y\left(t_{0}\right)=\max \left\{1,\left\|h_{\varepsilon_{0}}\right\|_{H^{1}}^{2}\right\} .
\end{aligned}
$$

Solving for $z(t)$,

$$
\left\|h_{\varepsilon}(\cdot, t)\right\|_{H^{1}}^{2} \leq y(t) \leq z(t)=\left[z\left(t_{0}\right)^{(1-2 m) / 2}-(2 m-1) C_{a}\left(t-t_{0}\right)\right]^{2 /(1-2 m)}
$$

for all

$$
t_{0} \leq t<t_{0}+\frac{1}{C_{a}(2 m-1)} \min \left\{1,\left\|h_{\varepsilon 0}\right\|_{H^{1}}^{1-2 m}\right\}=: t_{0}+T_{m \varepsilon} .
$$

Specifically, $\left\|h_{\varepsilon}(\cdot, t)\right\|_{H^{1}}$ is bounded for all $0 \leq t<T_{m \varepsilon}$. The second $H^{1}$ bound (8) is obtained by integrating inequality (9) in time.

Lemma 1 provides a priori bounds for $h_{\varepsilon}$ on the time interval $\left[0, T_{m \varepsilon}\right)$. Our next goal is to prove that as $\varepsilon \rightarrow 0$, the approximate solutions $h_{\varepsilon}$ converge to a weak solution of the original problem. Because $\left\|h_{\varepsilon 0}\right\|_{H^{1}} \rightarrow\left\|h_{0}\right\|_{H^{1}}$ as $\varepsilon \rightarrow 0$, it is clear that there exists an $\varepsilon_{0}$ such that for $\varepsilon<\varepsilon_{0}$, all the approximate solutions will be defined on the time interval $\left[0, T_{0}\right]$, where

$$
T_{0}:=\frac{1}{2} \frac{1}{C_{a}(2 m-1)} \min \left\{1,\left\|h_{0}\right\|_{H^{1}}^{1-2 m}\right\} .
$$

This allows us to define our weak solution on the time interval $\left[0, T_{0}\right]$. Clearly, the larger the $H^{1}$ norm of $h_{0}$, the smaller $T_{0}$ is.

In order to make the a priori bounds of Lemma 1 independent of $\varepsilon$, we need to remove the $\varepsilon$-dependence of righthand sides of inequalities (7) and (8). This $\varepsilon$-dependence appears through the presence of $\left\|h_{\varepsilon 0}\right\|_{H^{1}}$; we remove it by proving

$$
\left\|h_{0}\right\|_{H^{1}}^{2} \leq\left\|h_{\varepsilon 0}\right\|_{H^{1}}^{2} \leq\left(\left\|h_{0}\right\|_{H^{1}}+\sqrt{2} \varepsilon_{0}^{\vartheta}\right)^{2} .
$$

The first inequality is trivial. The second inequality follows from the Schwarz inequality and the specific $a$-dependence of $\delta(\varepsilon)$ :

$$
\left\|h_{\varepsilon 0}\right\|_{H^{1}}^{2}=\left\|h_{0}\right\|_{H^{1}}^{2}+2 \delta \int_{-a}^{a} h_{0}+2 a \delta^{2} \leq\left(\left\|h_{0}\right\|_{H^{1}}+\sqrt{2} \varepsilon^{\vartheta}\right)^{2} \leq\left(\left\|h_{0}\right\|_{H^{1}}+\sqrt{2} \varepsilon_{0}^{\vartheta}\right)^{2} .
$$

We now use the a priori $H^{1}$ bounds to prove further a priori bounds, also needed to pass to the $\varepsilon \rightarrow 0$ limit: 
Proposition 1. (Existence of approximate periodic solutions) Let $h_{0} \geq 0, h_{0} \in$ $H^{1}([-a, a]), T_{0}$ as defined in (12), and $m \geq 3$. Then the approximate equation (6) has a unique positive smooth solution $h_{\varepsilon}$ on the interval $\left[0, T_{0}\right]$. The approximate solution is positive with pointwise lower bound

$$
0<M_{\varepsilon}\left(T_{0},\left\|h_{0}\right\|_{H^{1}}\right) \leq h_{\varepsilon}(x, t) \quad t \in\left[0, T_{0}\right] .
$$

Moreover, for all $-\frac{1}{2}<s<1$, there are constants $C$ and $D$ independent of $\varepsilon$, dependent on $a, m, s, T_{0}$, and $\left\|h_{0}\right\|_{H^{1}}$ such that:

$$
\begin{aligned}
& \int_{0}^{T_{0}} \int_{-a}^{a}\left(h_{\varepsilon}^{s / 2+1}\right)_{x x}^{2}(x, t) d x d t \leq C T_{0}+D, \\
& \int_{0}^{T_{0}} \int_{-a}^{a}\left(h_{\varepsilon}^{s / 4+1 / 2}\right)_{x}^{4}(x, t) d x d t \leq C T_{0}+D,
\end{aligned}
$$

where

$$
\begin{aligned}
& \left\|h_{\varepsilon}\left(\cdot, T_{0}\right)\right\|_{H^{1}} \leq F_{m}\left(T_{0},\left\|h_{0}\right\|_{H^{1}}\right)<\infty, \\
& \left\|h_{\varepsilon}\left(\cdot, T_{0}\right)\right\|_{H^{1}}^{2} \leq\left\|h_{\varepsilon 0}\right\|_{H^{1}}^{2} e^{C \int_{0}^{T_{0}}\left\|h_{\varepsilon}(\cdot, \tau)\right\|_{L^{\infty}+\| h_{\varepsilon}(\cdot, \tau)} \|_{L^{\infty}}^{2 m-1} d \tau} \text {, } \\
& \mathcal{E}_{\varepsilon}\left(T_{0}\right) \leq \mathcal{E}_{\varepsilon}(0), \\
& \int_{0}^{T_{0}} \mathcal{E}_{\varepsilon}(t) d t \leq \mathcal{F}_{\varepsilon}(0) T_{0},
\end{aligned}
$$

$$
\mathcal{E}_{\varepsilon}(t):=\frac{1}{2} \int_{-a}^{a} h_{\varepsilon x}^{2}(x, t) d x-\int_{-a}^{a} \tilde{G}_{\varepsilon}\left(h_{\varepsilon}(x, t)\right) d x
$$

with $\tilde{G}_{\varepsilon}^{\prime \prime}(y)=g_{\varepsilon}(y) / f_{\varepsilon}(y)$.

Bounds (15) and (16) will be used to permit us to continue the solution in time. Bounds (17) and (18) will be used to prove the second-moment inequality.

Sketch of proof. The proof follows the arguments of earlier papers, in particular $[1,5,12,13]$. Bernis and Friedman [5] proved the short-time existence of a unique positive smooth solution for the equation $h_{\varepsilon t}=-\left(f_{\varepsilon}\left(h_{\varepsilon}\right) h_{\varepsilon x x x}\right)_{x}$. Their methods apply here to prove existence of a unique positive smooth solution for equation $(6)$ on a time interval $[0, \sigma]$. Lemma 1 gives an a priori bound of the $H^{1}$ norm of the solution $h_{\varepsilon}$ on the parabolic cylinder $Q_{T_{0}}$. Therefore, the solutions are a priori $C^{1 / 2}$ in space, with a Hölder constant independent of $\varepsilon$. In Appendix A, we prove that the $H^{1}$ bound gives an a priori bound on the $L^{2}$ norm of the flux on $Q_{T_{0}}$. This, combined with an argument found in Bernis and Friedman, shows the solutions are a priori $C^{1 / 8}$ in time, with a Hölder constant independent of $\varepsilon$.

To continue the solution to time $T_{0}$, one uses this a priori Hölder continuity and an a priori pointwise lower bound that holds up to time $T_{0}$. The a priori 
pointwise lower bound on [0, $T_{0}$ ] is found as follows. Following [5], define $G_{\varepsilon}(y)$ so that $G_{\varepsilon}^{\prime \prime}(y)=1 / f_{\varepsilon}(y)$. Since $T_{0}<T_{m \varepsilon}$, for all $t \leq T_{0}$,

$$
\begin{aligned}
& \frac{d}{d t} \int_{-a}^{a} G_{\varepsilon}\left(h_{\varepsilon}(x, t)\right) d x \\
& \quad=-\int_{-a}^{a} h_{\varepsilon x x}^{2}(x, t) d x+\int_{-a}^{a} \frac{g_{\varepsilon}\left(h_{\varepsilon}(x, t)\right)}{f_{\varepsilon}\left(h_{\varepsilon}(x, t)\right)} h_{\varepsilon x}^{2}(x, t) d x \\
& \quad \leq-\int_{-a}^{a} h_{\varepsilon x x}^{2}(x, t) d x+\left\|\frac{g_{\varepsilon}\left(h_{\varepsilon}(\cdot, t)\right)}{f_{\varepsilon}\left(h_{\varepsilon}(\cdot, t)\right)}\right\|_{L^{\infty}} \int_{-a}^{a} h_{\varepsilon x}^{2}(x, t) d x \\
& \quad \leq-\int_{-a}^{a} h_{\varepsilon x x}^{2}(x, t) d x+c\left(\left\|h_{\varepsilon 0}\right\|_{H^{1}}, m, T_{0}\right) .
\end{aligned}
$$

At step (19), we used the a priori $H^{1}$ bound (7) to find a uniform bound for $\left\|h_{\varepsilon}\right\|_{H^{1}}$ on $\left[0, T_{0}\right]$. Integrating,

$$
\begin{aligned}
& \int_{-a}^{a} G_{\varepsilon}\left(h_{\varepsilon}(x, t)\right) d x \\
& \leq \int_{-a}^{a} G_{\varepsilon}\left(h_{\varepsilon 0}(x)\right) d x+t c\left(\left\|h_{\varepsilon 0}\right\|_{H^{1}}, m, T_{0}\right) \leq C t+D,
\end{aligned}
$$

and

$$
\begin{aligned}
& \int_{0}^{T_{0}} \int_{-a}^{a} h_{\varepsilon x x}^{2}(x, t) d x d t \\
& \quad \leq \int_{-a}^{a} G_{\varepsilon}\left(h_{\varepsilon 0}(x)\right) d x+T_{0} C\left(\left\|h_{\varepsilon 0}\right\|_{H^{1}}, m, T_{0}\right) \leq C T_{0}+D
\end{aligned}
$$

Because $h_{\varepsilon 0}$ converges strongly to $h_{0}$ in $H^{1}$, the constants $C$ and $D$ are independent of $\varepsilon$. Because $\delta(\varepsilon)<\varepsilon^{1 / 2}$ in (6), there is a uniform-in- $\varepsilon$ bound on $\int G_{\varepsilon}\left(h_{\varepsilon 0}\right)$, and the constant $D$ is finite. Bound (21) is the bound (13) for $s=0$.

As in [5], this uniform bound on $\int G_{\varepsilon}\left(h_{\varepsilon}\right)$, combined with solutions being $C^{1 / 2}$ in space and $C^{1 / 8}$ in time, implies an a priori pointwise lower bound for $h_{\varepsilon}$ for all $t \leq T_{0}$. One can now continue the solution up to time $T_{0}$, finishing the existence part of the proof.

We now prove the approximate solution satisfies the bounds (13) and (14). Following [10], we define $G_{\varepsilon}^{s}$ where $G_{\varepsilon}^{s^{\prime \prime}}(y)=y^{s} / f_{\varepsilon}(y)$. The nonlinear energy satisfies 


$$
\begin{aligned}
\frac{d}{d t} \int_{-a}^{a} & G_{\varepsilon}^{s}\left(h_{\varepsilon}(x, t)\right) d x \\
=- & \frac{s(1-s)}{3} \int_{-a}^{a} h_{\varepsilon}^{s-2}(x, t) h_{\varepsilon x}^{4}(x, t) d x \\
& \quad-\int_{-a}^{a} h_{\varepsilon}^{s}(x, t) h_{\varepsilon x x}^{2}(x, t) d x \\
& +\int_{-a}^{a} h_{\varepsilon}^{s}(x, t) \frac{g_{\varepsilon}\left(h_{\varepsilon}(x, t)\right)}{f_{\varepsilon}\left(h_{\varepsilon}(x, t)\right)} h_{\varepsilon x}^{2}(x, t) d x \\
\leq- & \frac{s(1-s)}{3} \int_{-a}^{a} h_{\varepsilon}^{s-2}(x, t) h_{\varepsilon x}^{4}(x, t) d x \\
& \quad-\int_{-a}^{a} h_{\varepsilon}^{s}(x, t) h_{\varepsilon x x}^{2}(x, t) d x \\
& +C\left\|h_{\varepsilon}(\cdot, t)\right\|_{L^{\infty}}^{s}\left(1+\left\|h_{\varepsilon}(\cdot, t)\right\|_{L^{\infty}}^{m-1}\right) \int_{-a}^{a} h_{\varepsilon x}^{2}(x, t) d x .
\end{aligned}
$$

If $0<s<1$, then

$\int_{0}^{T_{0}} \int_{-a}^{a} h_{\varepsilon}^{s-2}(x, t) h_{\varepsilon x}^{4}(x, t) d x d t \quad$ and $\quad \int_{0}^{T_{0}} \int_{-a}^{a} h_{\varepsilon}^{s}(x, t) h_{\varepsilon x x}^{2}(x, t) d x d t$

are bounded above by

$$
c \int_{-a}^{a} G_{\varepsilon}^{s}\left(h_{\varepsilon 0}(x)\right) d x+T_{0} c\left(\left\|h_{\varepsilon 0}\right\|_{H^{1}}, m, T_{0}\right) \leq C T_{0}+D .
$$

The constant $D$ is finite and independent of $\varepsilon$, since $\delta(\varepsilon)<\varepsilon^{2 / 5}$ in (6), making $\int G_{\varepsilon}^{s}\left(h_{\varepsilon 0}\right)$ finite and bounded independent of $\varepsilon$. Also, $C$ is independent of $\varepsilon$.

We obtain the bounds for $-\frac{1}{2}<s<0$ as follows. First, note that

$$
\begin{aligned}
\frac{1-s}{3} \int_{-a}^{a} h_{\varepsilon}{ }^{s-2} h_{\varepsilon x}^{4} d x & =\int_{-a}^{a} h_{\varepsilon}^{s-1} h_{\varepsilon x}^{2} h_{\varepsilon x x} d x \\
& \leq \sqrt{\int_{-a}^{a} h_{\varepsilon}^{s-2} h_{\varepsilon x}^{2} d x} \sqrt{\int_{-a}^{a} h_{\varepsilon}^{s} h_{\varepsilon x x}^{2} d x}
\end{aligned}
$$

hence for $s<1$,

$$
\int_{-a}^{a} h_{\varepsilon}^{s-2}(x, t) h_{\varepsilon_{x}}^{4}(x, t) d x \leq \frac{9}{(1-s)^{2}} \int_{-a}^{a} h_{\varepsilon}^{s}(x, t) h_{\varepsilon x x}^{2}(x, t) d x .
$$


Using the identity (22), for $s<0$

$$
\begin{aligned}
\frac{d}{d t} \int_{-a}^{a} G_{\varepsilon}^{s}\left(h_{\varepsilon}(x, t)\right) d x \leq & \left(\frac{s(s-1)}{3} \frac{9}{(s-1)^{2}}-1\right) \int_{-a}^{a} h_{\varepsilon}^{s}(x, t) h_{\varepsilon x x}^{2}(x, t) d x \\
& +\int_{-a}^{a} h_{\varepsilon}^{s}(x, t) \frac{g_{\varepsilon}\left(h_{\varepsilon}(x, t)\right)}{f_{\varepsilon}\left(h_{\varepsilon}(x, t)\right)} h_{\varepsilon x}^{2}(x, t) d x \\
= & \frac{2 s+1}{s-1} \int_{-a}^{a} h_{\varepsilon}^{s}(x, t) h_{\varepsilon x x}^{2}(x, t) d x \\
& +\int_{-a}^{a} h_{\varepsilon}^{s}(x, t) \frac{g_{\varepsilon}\left(h_{\varepsilon}(x, t)\right)}{f_{\varepsilon}\left(h_{\varepsilon}(x, t)\right)} h_{\varepsilon x}^{2}(x, t) d x .
\end{aligned}
$$

Proceeding as before, for $-\frac{1}{2}<s<0$

$$
\int_{0}^{T_{0}} \int_{-a}^{a} h_{\varepsilon}^{s}(x, t) h_{\varepsilon x x}^{2}(x, t) d x d t \leq C T_{0}+D
$$

where $C$ and $D$ are independent of $\varepsilon$. The bound (23) then gives an upper bound independent of $\varepsilon$ for

$$
\int_{0}^{T_{0}} \int_{-a}^{a} h_{\varepsilon}^{s-2}(x, t) h_{\varepsilon x}^{4}(x, t) d x d t \leq C T_{0}+D
$$

This bound also holds for the $s=0$ case, by combining the bound (21) with (23). Because $h_{\varepsilon}$ is smooth, the bounds (13) and (14) follow immediately.

We finish by proving bounds (15-18). Bounds (15) and (16) follow immediately from Lemma 1 . Bounds (17) and (18) follow from identity

$$
\begin{aligned}
\frac{d}{d t} \mathcal{E}_{\varepsilon}(t)=-\int_{-a}^{a} f_{\varepsilon}\left(h_{\varepsilon}(x, t)\right)[ & h_{\varepsilon x x x}(x, t) \\
& \left.+\frac{g_{\varepsilon}\left(h_{\varepsilon}(x, t)\right)}{f_{\varepsilon}\left(h_{\varepsilon}(x, t)\right)} h_{\varepsilon x}(x, t)\right]^{2} d x \leq 0 .
\end{aligned}
$$

We use this identity in Appendix A to prove the flux is in $L^{2}\left(Q_{T_{0}}\right)$.

The entropies $\int G_{\varepsilon}\left(h_{\varepsilon}\right)$ and $\int G_{\varepsilon}^{s}\left(h_{\varepsilon}\right)$ are by now standard in the literature $[1,5,11,12,13]$ and the energy $\mathcal{E}_{\varepsilon}(t)$ is natural as well $[13,16,22,34]$. In this sense, Proposition 1 is standard. The only new aspect is choosing $\delta(\varepsilon)$ to ensure the a priori bounds depend on the interval size only through $\left\|h_{0}\right\|_{H^{1}(-a, a)}$. For compactly supported initial data, we will later remove this $a$-dependence since $\left\|h_{0}\right\|_{H^{1}(-a, a)}=\left\|h_{0}\right\|_{H^{1}(\mathbb{R})}$ for sufficiently large intervals. This will also allow us to take the a priori bounds (13-18) independent of $a$. 
3.2.1. The $\varepsilon \rightarrow 0$ limit. We now prove that the approximate solutions of Proposition 1 have a subsequence that converges to a nonnegative periodic weak solution in the sense of distributions:

$$
\begin{gathered}
-\int_{0}^{T_{0}} \int_{-a}^{a} h(x, t) \varphi_{t}(x, t) d x d t+\int_{-a}^{a} h\left(x, T_{0}\right) \varphi\left(x, T_{0}\right) d x \\
\quad-\int_{-a}^{a} h(x, 0) \varphi(x, 0) d x \\
=\int_{0}^{T_{0}} \int_{-a}^{a} \varphi_{x x x}(x, t) h(x, t) h_{x}(x, t) d x d t \\
\quad-\frac{1}{m+1} \int_{0}^{T_{0}} \int_{-a}^{a} \varphi_{x x}(x, t) h^{m+1}(x, t) d x d t \\
+\frac{3}{2} \int_{0}^{T_{0}} \int_{-a}^{a} \varphi_{x x}(x, t) h_{x}^{2}(x, t) d x d t .
\end{gathered}
$$

Theorem 1. (Existence of nonnegative periodic solutions) Let $h_{0} \geq 0, h_{0} \in$ $H^{1}([-a, a])$, and $T_{0}$ be as defined in (12). Let $h_{\varepsilon}$ be the approximate solutions of Proposition 1 on the time interval $\left[0, T_{0}\right]$. Then there exists a subsequence of $\left\{h_{\varepsilon}\right\}$, which converges pointwise uniformly on

$$
Q_{T_{0}}=[-a, a] \times\left[0, T_{0}\right]
$$

and weakly in

$$
L^{2}\left(0, T_{0} ; H^{2}([-a, a])\right) \cap L^{\infty}\left(0, T_{0} ; H^{1}([-a, a])\right)
$$

to a nonnegative periodic solution, $h$, in the sense of distributions (25). Furthermore, $h$ inherits the bounds (13-18) of Proposition 1.

Proof. By the uniform $C^{1 / 2,1 / 8}$ bound on the approximate solutions, we can apply the Arzela-Ascoli theorem. Hence, the family $\left\{h_{\varepsilon}\right\}$ has a subsequence that converges uniformly on $Q_{T_{0}}$ to a limit $h$.

Taking $s=0$ in bound (13), we have that $h_{\varepsilon}$ is uniformly bounded in $L^{2}\left(0, T ; H^{2}([-a, a])\right)$. Furthermore $d h_{\varepsilon} / d t$ is uniformly bounded in $H^{-1}([-a, a])$ and therefore $h_{\varepsilon}$ is uniformly bounded in $\operatorname{Lip}\left(0, T ; H^{-1}([-a, a])\right)$. The Lions-Aubin lemma applies and we can refine the subsequence so that in addition to converging pointwise uniformly to $h$, it also converges strongly in $L^{2}\left(0, T ; H^{1}([-a, a])\right)$. This then implies $h_{\varepsilon x}$ converges strongly to $h_{x}$ in $L^{2}\left(Q_{T_{0}}\right)$. Each approximate solution satisfies equation (2) in the sense of distributions: 


$$
\begin{aligned}
-\int_{0}^{T_{0}} \int_{-a}^{a} & h_{\varepsilon}(x, t) \varphi_{t}(x, t) d x d t \\
& +\int_{-a}^{a} h_{\varepsilon}\left(x, T_{0}\right) \varphi\left(x, T_{0}\right) d x-\int_{-a}^{a} h_{\varepsilon}(x, 0) \varphi(x, 0) d x \\
=\int_{0}^{T_{0}} & \int_{-a}^{a} \varphi_{x x x}(x, t) f_{\varepsilon}\left(h_{\varepsilon}(x, t)\right) h_{\varepsilon x}(x, t) d x d t \\
& +\frac{3}{2} \int_{0}^{T_{0}} \int_{-a}^{a} \varphi_{x x}(x, t) f_{\varepsilon}^{\prime}\left(h_{\varepsilon}(x, t)\right) h_{\varepsilon x}^{2}(x, t) d x d t \\
& +\frac{1}{2} \int_{0}^{T_{0}} \int_{-a}^{a} \varphi_{x}(x, t) f_{\varepsilon}^{\prime \prime}\left(h_{\varepsilon}(x, t)\right) h_{\varepsilon x}^{3}(x, t) d x d t \\
& -\int_{0}^{T_{0}} \int_{-a}^{a} \varphi_{x x}(x, t) \tilde{g}_{\varepsilon}\left(h_{\varepsilon}(x, t)\right) d x d t,
\end{aligned}
$$

where $\tilde{g}_{\varepsilon}^{\prime}(y)=g_{\varepsilon}(y)$.

We now prove that, as $\varepsilon \rightarrow 0$, each of the above terms converges to the appropriate term of equation (25). First, the a priori $H^{1}$ bound, $\left\|h_{\varepsilon}(\cdot, t)\right\|_{\infty} \leq M$, and the pointwise uniform convergence of $h_{\varepsilon} \rightarrow h$ imply that $g_{\varepsilon}\left(h_{\varepsilon}\right) \rightarrow h^{m+1}$ / $(m+1)$ and hence

$$
\begin{aligned}
\int_{0}^{T_{0}} \int_{-a}^{a} h_{\varepsilon}(x, t) \varphi_{t}(x, t) d x d t & \rightarrow \int_{0}^{T_{0}} \int_{-a}^{a} h(x, t) \varphi_{t}(x, t) d x d t, \\
\int_{-a}^{a} h_{\varepsilon}\left(x, T_{0}\right) \varphi\left(x, T_{0}\right) d x & \rightarrow \int_{-a}^{a} h\left(x, T_{0}\right) \varphi\left(x, T_{0}\right) d x, \\
\int_{-a}^{a} h_{\varepsilon}(x, 0) \varphi(x, 0) d x & \rightarrow \int_{-a}^{a} h(x, 0) \varphi(x, 0) d x, \\
\int_{0}^{T_{0}} \int_{-a}^{a} \varphi_{x x}(x, t) \tilde{g}_{\varepsilon}\left(h_{\varepsilon}(x, t)\right) d x d t & \rightarrow \frac{1}{m+1} \int_{0}^{T_{0}} \int_{-a}^{a} \varphi_{x x}(x, t) h^{m+1}(x, t) d x d t .
\end{aligned}
$$

Refining to a subsequence that converges strongly to $h_{x}$ in $L^{2}\left(Q_{T_{0}}\right)$ and using that $f_{\varepsilon}\left(h_{\varepsilon}\right)$ converges uniformly to $h$ (see appendix of [12]), we have

$$
\begin{aligned}
\int_{0}^{T_{0}} \int_{-a}^{a} \varphi_{x x x}(x, t) f_{\varepsilon}\left(h_{\varepsilon}(x, t)\right) & h_{\varepsilon x}(x, t) d x d t \\
& \rightarrow \int_{0}^{T_{0}} \int_{-a}^{a} \varphi_{x x x}(x, t) h(x, t) h_{x}(x, t) d x d t .
\end{aligned}
$$

We now prove that 


$$
\begin{aligned}
\int_{0}^{T_{0}} \int_{-a}^{a} \varphi_{x x}(x, t) f_{\varepsilon}^{\prime}\left(h_{\varepsilon}(x, t)\right) h_{\varepsilon x}^{2}(x, t) d x d t & \\
& \rightarrow \int_{0}^{T_{0}} \int_{-a}^{a} \varphi_{x x}(x, t) h_{x}^{2}(x, t) d x d t .
\end{aligned}
$$

First write

$$
\begin{aligned}
& \int_{0}^{T_{0}} \int_{-a}^{a} \varphi_{x x}(x, t) f_{\varepsilon}^{\prime}\left(h_{\varepsilon}(x, t)\right) h_{\varepsilon x}^{2}(x, t) d x d t \\
& \quad=\iint_{h>\mu} \varphi_{x x}(x, t) f_{\varepsilon}^{\prime}\left(h_{\varepsilon}(x, t)\right) h_{\varepsilon x}^{2}(x, t) d x d t \\
& \quad+\iint_{h \leq \mu} \varphi_{x x}(x, t) f_{\varepsilon}^{\prime}\left(h_{\varepsilon}(x, t)\right) h_{\varepsilon_{x}}^{2}(x, t) d x d t=I_{1}+I_{2} .
\end{aligned}
$$

Note that

$$
\begin{aligned}
\left|I_{2}\right| & \leq C\left\|f_{\varepsilon}^{\prime}\left(h_{\varepsilon}\right) h_{\varepsilon}^{(2-s) / 2}\right\|_{L^{2}(\{h<\mu\})}\left\|\left(h_{\varepsilon}^{s / 4+1 / 2}\right)_{x}\right\|_{L^{4}\left(Q_{T_{0}}\right)}^{2} \\
& \leq C\left\|f_{\varepsilon}^{\prime}\left(h_{\varepsilon}\right) h_{\varepsilon}^{(2-s) / 2}\right\|_{L^{2}(\{h<\mu\})}
\end{aligned}
$$

for any $-\frac{1}{2}<s<1$. Taking $s=-\frac{2}{5}$, as $\varepsilon \rightarrow 0, f_{\varepsilon}^{\prime}(y) y^{6 / 5}$ converges uniformly to $y^{6 / 5}$ on $[0, \mu]$ by [12, Lemma A.5]. Therefore $\left\|f_{\varepsilon}^{\prime}\left(h_{\varepsilon}\right) h_{\varepsilon}{ }^{6 / 5}\right\|_{L^{2}(\{h<\mu\})} \leq C \mu^{6 / 5}$, and taking $\mu \rightarrow 0$ yields $I_{2} \rightarrow 0$, as desired.

We now turn to $I_{1}$, the integral over $\{h>\mu\}$. By uniform parabolicity, $h_{\varepsilon x} \rightarrow h_{x}$ pointwise uniformly on the set $\{h>\mu\}$ as $\varepsilon \rightarrow 0$. Also $f^{\prime}\left(h_{\varepsilon}\right) \rightarrow 1$ on $\{h>\mu\}$. It follows that, as $\varepsilon \rightarrow 0$,

$$
I_{1} \rightarrow \iint_{h>\mu} \varphi_{x x}(x, t) h_{x}^{2}(x, t) d x d t .
$$

To take $\mu \rightarrow 0$, we first introduce the set

$$
\mathcal{P}=\overline{Q_{T}}-\{\{h=0\} \cup\{t=0\}\} .
$$

We see that

$$
I_{1} \rightarrow \iint_{\mathcal{P}} \varphi_{x x}(x, t) h_{x}^{2}(x, t) d x d t
$$

after taking $\mu$ to zero. It remains to prove that

$$
\iint_{\mathcal{P}} \varphi_{x x}(x, t) h_{x}^{2}(x, t) d x d t=\int_{0}^{T_{0}} \int_{-a}^{a} \varphi_{x x}(x, t) h_{x}^{2}(x, t) d x d t .
$$

Proving this equality involves a new argument, not used in previous articles on these equations. First, for $0<t \leq T_{0}$, we define time-slices of $\mathcal{P}$ :

$$
\mathcal{P}_{t}:=\{x \mid h(x, t)>0\} \subset[-a, a] .
$$


$\mathcal{P}$ is a measurable set, $h_{x}$ is jointly measurable in $x$ and $t$ (since $h_{x} \in L^{2}\left(Q_{T_{0}}\right)$ ), and $\chi_{\mathcal{P}} \varphi_{x x} h_{x}^{2} \in L^{1}\left(Q_{T_{0}}\right)$, allowing us to use Fubini's theorem to verify the second equality:

$$
\begin{aligned}
\iint_{\mathcal{P}} \varphi_{x x}(x, t) h_{x}^{2}(x, t) d x d t & =\iint_{Q_{T_{0}}} x_{\mathcal{P}}(x, t) \varphi_{x x}(x, t) h_{x}^{2}(x, t) d x d t \\
& =\int_{0}^{T_{0}} \int_{-a}^{a} x_{\mathcal{P}_{t}}(x) \varphi_{x x}(x, t) h_{x}^{2}(x, t) d x d t .
\end{aligned}
$$

Here $\chi_{\mathcal{P}}$ is the characteristic function of $\mathcal{P} \subset Q_{T_{0}}$ and $\chi_{\mathcal{P}_{t}}$ is the characteristic function of $\mathcal{P}_{t} \subset[-a, a]$. In the above, we used that $h_{0} \in H^{1}([-a, a])$, making the integrand finite at $t=0$. This allows us to extend the integral from $\mathcal{P}$ to $\overline{Q_{T}}-\{h=0\}$, as desired.

Now, we use the following lemma from Gilbarg \& Trudinger [20, page 152]:

Lemma 7.7. Let $h \in W^{1}(\Omega)$ (i.e., $h$ has a weak derivative.) Then $D h=0$ almost everywhere on any set where $h$ is constant.

Recalling that $h \geq 0$, we apply the lemma to the set where $h=0$. Therefore

$$
\int_{-a}^{a} x_{\mathcal{P}_{t}}(x) \varphi_{x x}(x, t) h_{x}(x, t) d x=\int_{-a}^{a} \varphi_{x x}(x, t) h_{x}(x, t) d x .
$$

Integrating in time yields the desired result:

$$
\begin{aligned}
\iint_{\mathcal{P}} \varphi_{x x}(x, t) h_{x}^{2}(x, t) d x d t & =\int_{0}^{T_{0}} \int_{-a}^{a} x_{\mathcal{P}_{t}}(x) \varphi_{x x}(x, t) h_{x}^{2}(x, t) d x d t \\
& =\int_{0}^{T_{0}} \int_{-a}^{a} \varphi_{x x}(x, t) h_{x}^{2}(x, t) d x d t .
\end{aligned}
$$

Finally, we show that $\iint \varphi_{x} f_{\varepsilon}^{\prime \prime}\left(h_{\varepsilon}\right) h_{\varepsilon x}^{3}$ converges to zero as $\varepsilon \rightarrow 0$. This step is special to the case $f(h)=h$ in (1). First,

$$
\begin{aligned}
I & =\iint \varphi_{x}(x, t) f_{\varepsilon}^{\prime \prime}\left(h_{\varepsilon}(x, t)\right) h_{\varepsilon x}^{3}(x, t) d x d t \\
& =\left(\frac{12}{5}\right)^{3} \iint \varphi_{x}(x, t) f_{\varepsilon}^{\prime \prime}\left(h_{\varepsilon}(x, t)\right) h_{\varepsilon}^{7 / 4}(x, t)\left(h_{\varepsilon}^{5 / 12}\right)_{x}^{3}(x, t) d x d t .
\end{aligned}
$$

Thus $|I|$ is bounded by

$$
\left(\frac{12}{5}\right)^{3}\left\|f_{\varepsilon}^{\prime \prime}\left(h_{\varepsilon}\right) h_{\varepsilon}{ }^{7 / 4}\right\|_{L^{\infty}}\left\|\left(h_{\varepsilon}^{5 / 12}\right)_{x}\right\|_{L^{4}}^{3}\left\|\varphi_{x}\right\|_{L^{4}} .
$$

In Appendix B, we prove that $\left\|f_{\varepsilon}^{\prime \prime}\left(h_{\varepsilon}\right) h_{\varepsilon}{ }^{7 / 4}\right\| \leq C \varepsilon^{1 / 4}$ for some constant $C$. Again taking $s=-\frac{1}{3}$ in (14), $\left\|\left(h_{\varepsilon}^{5 / 12}\right)_{x}\right\|_{L^{4}}$ is uniformly bounded. Because $\varphi$ is 
a smooth test function, $\left\|\varphi_{x}\right\|_{L^{4}}$ is bounded. It follows that $\iint \varphi_{x} f_{\varepsilon}^{\prime \prime}\left(h_{\varepsilon}\right) h_{\varepsilon x}^{3} \rightarrow 0$ as $\varepsilon \rightarrow 0$.

This proves that $h$ satisfies the PDE in the sense of distributions (5).

For a fixed $-\frac{1}{2}<s<0$, one can take a further refinement of the subsequence $\left\{h_{\varepsilon}\right\}$ to ensure that the limiting function $h$ inherits the bounds (13-14) for that value of $s$. A final refinement of the subsequence ensures the limiting function also inherits the bound (15). Then, this bound and uniform convergence of $h_{\varepsilon}$ guarantees the remaining bounds (16-18).

3.3. Finite speed of propagation for periodic nonnegative weak solutions. Following Bernis [3], we say that a solution has finite speed of propagation if it satisfies the following.

Definition 1. Let $h:[-a, a] \times\left[0, T_{0}\right] \rightarrow \mathbb{R}$ be a function such that $h(\cdot, 0)=$ $h_{0}$ in $(-a, a)$ with $h_{0}=0$ in some nonempty open subset $\omega=\left(b-r_{0}, b+r_{0}\right)$ of $(-a, a)$. The solution has finite speed of propagation if for each such subset $\omega$, there exists a time $T^{*}>0$ and two continuous functions on $\left(0, T^{*}\right)$ such that $b_{-}(t)<b_{+}(t), b_{-}(0)=b-r_{0}, b_{+}(0)=b+r_{0}$, and $h(\cdot, t) \equiv 0$ in $\left(b_{-}(t), b_{+}(t)\right)$ for all $t \in\left(0, T^{*}\right)$.

For each initially "dry" region $\omega$ there will be a time $T^{*}$ for which the region must persist (although it may shrink). As one would expect, the time $T^{*}$ is such that the smaller the initial diameter, $2 r_{0}$, the shorter the guaranteed time of persistence $T^{*}$. For our purposes, we are interested in the diameter of the support, as opposed to internal dry regions. We define the leftmost and rightmost contact lines as follows:

Definition 2. For each $t \in\left[0, T_{0}\right]$, we define

$$
\zeta_{L}(t)= \begin{cases}\inf \{x \in \operatorname{supp}\{h(\cdot, t)\} \mid x \geq-a\}, & \text { if the set is nonempty, } \\ -a & \text { otherwise. }\end{cases}
$$

We similarly define

$$
\zeta_{R}(t)= \begin{cases}\sup \{x \in \operatorname{supp}\{h(\cdot, t)\} \mid x \leq a\}, & \text { if the set is nonempty, } \\ a & \text { otherwise. }\end{cases}
$$

We prove the following proposition for finite speed of propagation:

Proposition 2. Assume $h_{0}$ is nonnegative, $h_{0} \in H^{1}(\mathbb{R})$, and $\operatorname{supp}\left\{h_{0}\right\} \subset$ $\left(-a_{0}, a_{0}\right)$, where $a_{0}<a$. Then the solution $h$ of Theorem 1 has finite speed of propagation as defined above. Furthermore, let $s \in\left(-\frac{1}{2}, 1\right), s \neq 0$. Then there exists a diameter $a_{*}=a_{*}\left(s, \varphi_{1}, m, h_{0}, a_{0}, T_{0}\right)$ such that, if the domain $(-a, a)$ is chosen with $a \geq a_{*}$, then

$$
-a<\zeta_{L}(T), \quad \zeta_{R}(T)<a, \quad \text { for all } T \in\left[0, T_{0}\right] .
$$


The diameter $a_{x}$ depends on a fixed test function, $\varphi_{1}$, which we describe below. Furthermore,

$$
\zeta_{R}(T)-\zeta_{R}(0) \leq A_{0} T^{\alpha}\left(\int_{0}^{T} \int_{\zeta_{R}(0)}^{a}\left(h^{(s+2) / 2}\right)_{x x}^{2} d x d t\right)^{\beta},
$$

where $A_{0}$ is a positive constant depending only on $s, m, h_{0}, T_{0}$, and $\varphi_{1}$ and

$$
\alpha=\frac{1+s}{5+4 s}, \quad \beta=\frac{1}{5+4 s} .
$$

The leftmost contact line satisfies an analogous bound.

We present the proof in Appendix C. The proof follows directly from the methods of Bernis [3]. The proof uses local energy methods. This involves introducing a cut-off function to localize the energy and then controlling the evolution of this localized energy. We take the cut-off function, $\xi=\varphi_{r}^{4}$, where

$$
\begin{aligned}
\varphi_{r}(x) & =r \varphi_{1}\left(\frac{x}{r}\right), \quad r \leq a, \varphi_{1} \geq 0, \\
\operatorname{supp}\left\{\varphi_{1}\right\} & =(-1,1), \quad \varphi_{1} \in C_{c}(\mathbb{R}) \cap C^{2}((-1,1)) .
\end{aligned}
$$

We now prove an analogue of Lemma 4.5 in [3]. This is the key lemma in proving the finite speed of propagation. It is here that we control the extra terms that arise from the second-order term in the evolution equation (Bernis considered $h_{t}=-\left(f(h) h_{x x x}\right)_{x}$. $)$

Lemma 2. Let $\xi$ be as in (26) and assume $-\frac{1}{2}<s<1, s \neq 0$. Then there exist constants $C, A_{1}$, and $A_{2}$ dependent on $s, \varphi_{1}, h_{0}, T_{0}$, and $m$, independent of $a$ and $\varepsilon$, such that for all $T \leq T_{0}$

$$
\begin{aligned}
& \frac{1}{s(s+1)} \int_{-a}^{a} \varphi_{r}^{4} h^{s+1}(x, T) d x+A_{1} \int_{0}^{T} \int_{-a}^{a} \varphi_{r}^{4}\left(h^{(s+2) / 2}\right)_{x x}^{2} d x d t \\
& \quad \leq e^{C s(s+1) T} \frac{1}{s(s+1)} \int_{-a}^{a} \varphi_{r}^{4} h_{0}^{s+1}(x) d x+A_{2} \int_{0}^{T} \int_{-r}^{r} h^{s+2} d x d t
\end{aligned}
$$

In fact, the lemma does not need that $\operatorname{supp}\left\{\varphi_{r}\right\}=(-r, r)$. The proof applies for any $\varphi_{r}$ that satisfies (26) with $\operatorname{supp}\left\{\varphi_{r}\right\} \subset(-a, a)$. The only change is that the upper bound in Lemma 2 would have an integral over $Q_{T} \cap \operatorname{supp}\left\{\varphi_{r}\right\}$.

The proof uses the following local calculus inequalities [3].

Lemma. Let $s \in \mathbb{R}, s \neq 1$, and let $v$ and $\xi$ be real-valued functions on $[-a, a]$ such that $v \in H^{2}(-a, a), v>0, \xi \in C^{1}([-a, a])$, and $\xi \geq 0$. Assume 
that $v(-a)=v(a), v_{x}(-a)=v_{x}(a)$, and $\xi(-a)=\xi(a)$. Then

$$
\begin{aligned}
\int_{-a}^{a} \xi(x) v^{s-2}(x) v_{x}^{4}(x) d x \leq & \frac{9}{(1-s)^{2}} \int_{-a}^{a} \xi(x) v^{s}(x) v_{x x}^{2}(x) d x \\
& +\frac{2}{1-s} \int_{-a}^{a} \xi^{\prime}(x) v^{s-1}(x) v_{x}^{3}(x) d x .
\end{aligned}
$$

Lemma. Under the above hypotheses, if in addition $s \leq 0$, then

$$
\begin{aligned}
& \int_{-a}^{a} \xi(x) v^{s}(x) v_{x x}^{2}(x) d x+\frac{s(1-s)}{3} \int_{-a}^{a} \xi(x) v^{s-2}(x) v_{x}^{4}(x) d x \\
& \quad \geq \frac{2 s+1}{1-s} \int_{-a}^{a} \xi(x) v^{s}(x) v_{x x}^{2}(x) d x+\frac{2}{3} s \int_{-a}^{a} \xi^{\prime}(x) v^{s-1}(x) v_{x}^{3}(x) d x .
\end{aligned}
$$

Proof of Lemma 2. We consider a localized energy: ${ }^{2}$

$$
\begin{aligned}
& \frac{d}{d t} \int_{-a}^{a} \xi(x) G_{\varepsilon}^{s}\left(h_{\varepsilon}(x, t)\right) d x \\
&=-\int_{-a}^{a} \xi^{\prime}(x) G_{\varepsilon}^{s^{\prime}}\left(h_{\varepsilon}(x, t)\right) f_{\varepsilon}^{\prime}\left(h_{\varepsilon}(x, t)\right) h_{\varepsilon x}(x, t) h_{\varepsilon x x}(x, t) d x \\
& \quad-2 \int_{-a}^{a} \xi^{\prime}(x) h_{\varepsilon}^{s}(x, t) h_{\varepsilon x}(x, t) h_{\varepsilon x x}(x, t) d x \\
&+\frac{s(s-1)}{3} \int_{-a}^{a} \xi(x) h_{\varepsilon}{ }^{s-2}(x, t) h_{\varepsilon x}^{4}(x, t) d x \\
&+ \frac{s}{3} \int_{-a}^{a} \xi^{\prime}(x) h_{\varepsilon}{ }^{s-1}(x, t) h_{\varepsilon x}^{3}(x, t) d x \\
&-\int_{-a}^{a} \xi^{\prime \prime}(x) G_{\varepsilon}^{s^{\prime}}\left(h_{\varepsilon}(x, t)\right) f_{\varepsilon}\left(h_{\varepsilon}(x, t)\right) h_{\varepsilon x x}(x, t) d x \\
&-\int_{-a}^{a} \xi(x) h_{\varepsilon}{ }^{s}(x, t) h_{\varepsilon x x}^{2}(x, t) d x \\
&+\int_{-a}^{a} \xi(x) h_{\varepsilon}{ }^{s}(x, t) \frac{g_{\varepsilon}\left(h_{\varepsilon}(x, t)\right)}{f_{\varepsilon}\left(h_{\varepsilon}(x, t)\right)} h_{\varepsilon x}^{2}(x, t) d x \\
&+\int_{-a}^{a} \xi^{\prime}(x) G_{\varepsilon}^{s^{\prime}}\left(h_{\varepsilon}(x, t)\right) g_{\varepsilon}\left(h_{\varepsilon}(x, t)\right) h_{\varepsilon x}(x, t) d x .
\end{aligned}
$$

\footnotetext{
${ }^{2}$ The second-to-last term above differs from the second-to-last term in inequality (3.19) in an earlier article of ours [13]: that article has a misprint.
} 
Using the definitions of $g_{\varepsilon}, f_{\varepsilon}$, and $G_{\varepsilon}^{s}$, one can find a constant $C$, depending only on $s$, such that

$$
\begin{array}{ll}
\left|G_{\varepsilon}^{s^{\prime}}(y) f_{\varepsilon}(y)\right| \leq C y^{1+s}, & \left|G_{\varepsilon}^{s^{\prime}}(y) f_{\varepsilon}^{\prime}(y)\right| \leq C y^{s}, \\
\left|G_{\varepsilon}^{s^{\prime}}(y) g_{\varepsilon}(y)\right| \leq C y^{s+1}\left(1+y^{m-1}\right), & \left|\frac{g_{\varepsilon}(y)}{f_{\varepsilon}(y)}\right| \leq C\left(1+y^{m-1}\right) .
\end{array}
$$

As in Bernis [3], one uses the bounds (28)-(29) and calculus inequalities to find for any $t \leq T_{0}$

$$
\begin{aligned}
\frac{d}{d t} \int_{-a}^{a} \xi(x) G_{\varepsilon}^{s}\left(h_{\varepsilon}(x, t)\right) d x+c_{1} \int_{-a}^{a} \xi(x) h_{\varepsilon}{ }^{s}(x, t) h_{\varepsilon x x}^{2}(x, t) d x \\
\quad+c_{2} \int_{-a}^{a} \xi(x) h_{\varepsilon}^{s-2}(x, t) h_{\varepsilon x}^{4}(x, t) d x \\
\leq C\left[\int_{-a}^{a}\left|\xi^{\prime \prime}(x) h_{\varepsilon}{ }^{s+1}(x, t) h_{\varepsilon x x}(x, t)\right| d x\right. \\
\quad+\int_{-a}^{a}\left|\xi^{\prime}(x) h_{\varepsilon}{ }^{s-1}(x, t) h_{\varepsilon x}^{3}(x, t)\right| d x \\
\quad+\int_{-a}^{a}\left|\xi^{\prime}(x) h_{\varepsilon}{ }^{s}(x, t) h_{\varepsilon x}(x, t) h_{\varepsilon x x}(x, t)\right| d x \\
\quad+\int_{-a}^{a}\left|\xi^{\prime}(x) h_{\varepsilon}{ }^{s+1}(x, t)\left(1+h_{\varepsilon}{ }^{m-1}(x, t)\right) h_{\varepsilon x}(x, t)\right| d x \\
\left.\quad+\int_{-a}^{a}\left|\xi(x) h_{\varepsilon}{ }^{s}(x, t)\left(1+h_{\varepsilon}{ }^{m-1}(x, t)\right) h_{\varepsilon x}^{2}(x, t)\right| d x\right] .
\end{aligned}
$$

The constants $c_{1}, c_{2}$, and $C$ depend on $s$ only.

From (26), there are constants $a_{1}$ and $a_{2}$ depending only on $\varphi_{1}$ such that

$$
\left|\xi^{\prime}(x)\right| \leq a_{1} \varphi_{r}^{3}(x), \quad\left|\xi^{\prime \prime}(x)\right| \leq a_{2} \varphi_{r}^{2}(x) .
$$

We now bound each of the terms on the right-hand side of inequality (30). For example, the third term can be bounded as follows:

$$
\begin{aligned}
& \int_{-a}^{a}\left|\xi^{\prime}(x) h_{\varepsilon}^{s}(x, t) h_{\varepsilon x}(x, t) h_{\varepsilon x x}(x, t)\right| d x \\
& \quad \leq a_{1} \int_{-a}^{a} \varphi_{r}^{3}(x) h_{\varepsilon}^{s}(x, t)\left|h_{\varepsilon x}(x, t) h_{\varepsilon x x}(x, t)\right| d x \\
& \quad \leq a_{1} \sqrt{\int_{-a}^{a} \varphi_{r}^{4} h_{\varepsilon}^{s} h_{\varepsilon x x}^{2} d x} \sqrt[4]{\int_{-a}^{a} \varphi_{r}^{4} h_{\varepsilon}^{s-2} h_{\varepsilon x}^{4} d x} \sqrt[4]{\int_{-r}^{r} h_{\varepsilon}^{s+2} d x} \\
& \quad \leq \delta \int_{-a}^{a} \varphi_{r}^{4} h_{\varepsilon}^{s} h_{\varepsilon x x}^{2} d x+\delta \int_{-a}^{a} \varphi_{r}^{4} h_{\varepsilon}^{s-2} h_{\varepsilon x}^{4} d x+C_{\delta} \int_{-r}^{r} h_{\varepsilon}^{s+2} d x .
\end{aligned}
$$


In the second step, we used the Schwarz inequality twice and the fact that $\operatorname{supp}\left\{\varphi_{r}\right\}=(-r, r)$. In the third step, we used the Young inequality twice. As in Bernis [3], the first and second terms can be bounded similarly. We now turn to the fourth and fifth terms; the terms that arise from the second-order term in the evolution equation. The fourth term is bounded by

$$
\begin{aligned}
& a_{1} \int_{-a}^{a} \varphi_{r}^{3} h_{\varepsilon}{ }^{s+1}\left(1+h_{\varepsilon}{ }^{m-1}\right)\left|h_{\varepsilon x}\right| d x \\
& \leq C\left(m, h_{0}, T_{0}\right) \int_{-a}^{a} \varphi_{r}^{3} h_{\varepsilon}{ }^{s+1}\left|h_{\varepsilon x}\right| d x \\
& \leq C\left(m, h_{0}, T_{0}\right)\left(\int_{-a}^{a} \varphi_{r}^{4} h_{\varepsilon}{ }^{s-2} h_{\varepsilon x}^{4} d x\right)^{1 / 4}\left(\int_{-a}^{a} \varphi_{r}^{8 / 3} h_{\varepsilon}{ }^{s+2} d x\right)^{3 / 4} \\
& \leq \delta \int_{-a}^{a} \varphi_{r}^{4} h_{\varepsilon}{ }^{s-2} h_{\varepsilon x}^{4} d x+C\left(\delta, m, h_{0}, T_{0}\right) \int_{-a}^{a} \varphi_{r}^{8 / 3} h_{\varepsilon}^{s+2} d x \\
& \leq \delta \int_{-a}^{a} \varphi_{r}^{4} h_{\varepsilon}{ }^{s-2} h_{\varepsilon x}^{4} d x \\
&+C\left(\delta, m, h_{0}, T_{0}\right)\left(\int_{-a}^{a} \varphi_{r}^{4} h_{\varepsilon}{ }^{s+1} d x\right)^{2 / 3}\left(\int_{-r}^{r} h_{\varepsilon}^{s+4} d x\right)^{1 / 3} \\
& \leq \delta \int_{-a}^{a} \varphi_{r}^{4} h_{\varepsilon}{ }^{s-2} h_{\varepsilon x}^{4} d x+C\left(\delta, m, h_{0}, T_{0}\right) \int_{-a}^{a} \varphi_{r}^{4} h_{\varepsilon}{ }^{s+1} d x \\
&+C\left(\delta, m, h_{0}, T_{0}\right) \int_{-r}^{r} h_{\varepsilon}{ }^{s+2} d x .
\end{aligned}
$$

At step (31), we used $m>1$ and the a priori bound on $\left\|h_{\varepsilon}\right\|_{\infty}$ of Lemma 1 . At step (32), we used Hölder's inequality with $p=4$ and $q=\frac{4}{3}$. At step (33), we used Young's inequality. At step (34) we used Hölder's inequality with $p=3 / 2$ and $q=3$. At step (35) we used Young's inequality and the a priori bound on $\left\|h_{\varepsilon}\right\|_{\infty}$.

The fifth term is bounded by

$$
\begin{aligned}
& \int_{-a}^{a} \varphi_{r}^{4} h_{\varepsilon}^{s}\left(1+h_{\varepsilon}{ }^{m-1}\right) h_{\varepsilon x}^{2} d x \\
& \quad \leq C\left(m, h_{0}, T_{0}\right) \int_{-a}^{a} \varphi_{r}^{4} h_{\varepsilon}^{s} h_{\varepsilon x}^{2} d x \\
& \quad \leq C\left(m, h_{0}, T_{0}\right) \sqrt{\int_{-a}^{a} \varphi_{r}^{4} h_{\varepsilon}{ }^{s-2} h_{\varepsilon x}^{4} d x} \sqrt{\int_{-a}^{a} \varphi_{r}^{4} h_{\varepsilon}^{s+2} d x} \\
& \quad \leq \delta \int_{-a}^{a} \varphi_{r}^{4} h_{\varepsilon}{ }^{s-2} h_{\varepsilon x}^{4} d x+C\left(\delta, m, h_{0}, T_{0}\right) \int_{-a}^{a} \varphi_{r}^{4} h_{\varepsilon}{ }^{s+2} d x \\
& \quad \leq \delta \int_{-a}^{a} \varphi_{r}^{4} h_{\varepsilon}{ }^{s-2} h_{\varepsilon x}^{4} d x+C\left(\delta, m, h_{0}, T_{0}\right) \int_{-a}^{a} \varphi_{r}^{4} h_{\varepsilon}{ }^{s+1} d x
\end{aligned}
$$


At step (36) we used $m>1$ and the a priori bound on $\left\|h_{\varepsilon}\right\|_{\infty}$. At step (37) we used Schwarz's inequality. At step (38) we used Young's inequality. At step (39) we used the a priori bound on $\left\|h_{\varepsilon}\right\|_{\infty}$.

Combining these bounds, and taking $\delta$ small, the bound (30) becomes

$$
\begin{gathered}
\frac{d}{d t} \int_{-a}^{a} \varphi_{r}^{4}(x) G_{\varepsilon}^{s}\left(h_{\varepsilon}(x, t)\right) d x+c_{1} \int_{-a}^{a} \varphi_{r}^{4}(x) h_{\varepsilon}^{s}(x, t) h_{\varepsilon x x}^{2}(x, t) d x \\
+c_{2} \int_{-a}^{a} \varphi_{r}^{4}(x) h_{\varepsilon}^{s-2}(x, t) h_{\varepsilon x}^{4}(x, t) d x \\
\quad \leq C_{1} \int_{-r}^{r} h_{\varepsilon}^{s+2}(x, t) d x+C_{2} \int_{-a}^{a} \varphi_{r}^{4}(x) h_{\varepsilon}{ }^{s+1}(x, t) d x
\end{gathered}
$$

The constants $c_{1}, c_{2}, C_{1}$, and $C_{2}$ depend on $s, \varphi_{1}, m, h_{0}$, and $T_{0}$ only. Using the chain rule,

$$
\begin{aligned}
\frac{d}{d t} \int_{-a}^{a} \varphi_{r}^{4}(x) G_{\varepsilon}^{s}\left(h_{\varepsilon}(x, t)\right) d x+c \int_{-a}^{a} & \varphi_{r}^{4}(x)\left(h_{\varepsilon}^{(s+2) / 2}\right)_{x x}^{2} d x \\
& \leq C \int_{-r}^{r} h_{\varepsilon}^{s+2} d x+C \int_{-a}^{a} \varphi_{r}^{4} h_{\varepsilon}{ }^{s+1} d x
\end{aligned}
$$

where $c$ and $C$ depend on $s, \varphi_{1}, m, h_{0}$, and $T_{0}$ only. We subtract $D \int \varphi_{r}^{4} G_{\varepsilon}^{s}\left(h_{\varepsilon}\right)$ from both sides of the inequality, where $D$ is a constant yet to be specified. Integrating,

$$
\begin{aligned}
& \int_{-a}^{a} \varphi_{r}^{4} G_{\varepsilon}^{s}\left(h_{\varepsilon}\left(x, T_{2}\right)\right) d x-e^{D\left(T_{2}-T_{1}\right)} \int_{-a}^{a} \varphi_{r}^{4} G_{\varepsilon}^{s}\left(h_{\varepsilon}\left(x, T_{1}\right)\right) d x \\
& \leq-c \int_{T_{1}}^{T_{2}} \int_{-a}^{a} e^{D\left(T_{2}-t\right)} \varphi_{r}^{4}\left(h_{\varepsilon}^{(s+2) / 2}\right)_{x x}^{2} d x d t+C \int_{T_{1}}^{T_{2}} \int_{-r}^{r} e^{D\left(T_{2}-t\right)} h_{\varepsilon}^{s+2} d x d t \\
& \quad+\int_{T_{1}}^{T_{2}} \int_{-a}^{a} e^{D\left(T_{2}-t\right)} \varphi_{r}^{4}\left(C h_{\varepsilon}^{s+1}-D G_{\varepsilon}^{s}\left(h_{\varepsilon}\right)\right) d x d t .
\end{aligned}
$$

We now pass to the limit in $\varepsilon$,

$$
\begin{array}{r}
\frac{1}{s(s+1)} \int_{-a}^{a} \varphi_{r}^{4} h^{s+1}\left(x, T_{2}\right) d x-e^{D\left(T_{2}-T_{1}\right)} \frac{1}{s(s+1)} \int_{-a}^{a} \varphi_{r}^{4} h^{s+1}\left(x, T_{1}\right) d x \\
\leq-c \int_{T_{1}}^{T_{2}} \int_{-a}^{a} e^{D\left(T_{2}-t\right)} \varphi_{r}^{4}\left(h^{(s+2) / 2}\right)_{x x}^{2} d x d t+C \int_{T_{1}}^{T_{2}} \int_{-r}^{r} e^{D\left(T_{2}-t\right)} h^{s+2} d x d t \\
+\int_{T_{1}}^{T_{2}} \int_{-a}^{a} e^{D\left(T_{2}-t\right)} \varphi_{r}^{4}\left(C-D \frac{1}{s(s+1)}\right) h^{s+1} d x d t .
\end{array}
$$


Taking $D=C s(s+1)$,

$$
\begin{array}{r}
\frac{1}{s(s+1)} \int_{-a}^{a} \varphi_{r}^{4} h^{s+1}\left(x, T_{2}\right) d x-e^{C s(s+1)\left(T_{2}-T_{1}\right)} \frac{1}{s(s+1)} \int_{-a}^{a} \varphi_{r}^{4} h^{s+1}\left(x, T_{1}\right) d x \\
\leq-c \int_{T_{1}}^{T_{2}} \int_{-a}^{a} e^{C s(s+1)\left(T_{2}-t\right)} \varphi_{r}^{4}\left(h^{(s+2) / 2}\right)_{x x}^{2} d x d t \\
+C \int_{T_{1}}^{T_{2}} \int_{-r}^{r} e^{C s(s+1)\left(T_{2}-t\right)} h^{s+2} d x d t .
\end{array}
$$

The sign of the exponents can be either positive or negative. If $-\frac{1}{2}<s<0$, then

$$
\begin{array}{r}
\frac{1}{s(s+1)} \int_{-a}^{a} \varphi_{r}^{4} h^{s+1}\left(x, T_{2}\right) d x-e^{C s(s+1)\left(T_{2}-T_{1}\right)} \frac{1}{s(s+1)} \int_{-a}^{a} \varphi_{r}^{4} h^{s+1}\left(x, T_{1}\right) d x \\
\leq-c e^{C s(s+1) T_{0}} \int_{T_{1}}^{T_{2}} \int_{-a}^{a} \varphi_{r}^{4}\left(h^{(s+2) / 2}\right)_{x x}^{2} d x d t+C \int_{T_{1}}^{T_{2}} \int_{-r}^{r} h^{s+2} d x d t .
\end{array}
$$

If $0<s<1$, then

$$
\begin{array}{r}
\frac{1}{s(s+1)} \int_{-a}^{a} \varphi_{r}^{4} h^{s+1}\left(x, T_{2}\right) d x-e^{C s(s+1)\left(T_{2}-T_{1}\right)} \frac{1}{s(s+1)} \int_{-a}^{a} \varphi_{r}^{4} h^{s+1}\left(x, T_{1}\right) d x \\
\leq-c \int_{T_{1}}^{T_{2}} \int_{-a}^{a} \varphi_{r}^{4}\left(h^{(s+2) / 2}\right)_{x x}^{2} d x d t+C e^{C s(s+1) T_{0}} \int_{T_{1}}^{T_{2}} \int_{-r}^{r} h^{s+2} d x d t .
\end{array}
$$

In both cases,

$$
\begin{aligned}
& \frac{1}{s(s+1)} \int_{-a}^{a} \varphi_{r}^{4} h^{s+1}\left(x, T_{2}\right) d x+A_{1} \int_{T_{1}}^{T_{2}} \int_{-a}^{a} \varphi_{r}^{4}\left(h^{(s+2) / 2}\right)_{x x}^{2} d x d t \\
& \leq e^{C s(s+1)\left(T_{2}-T_{1}\right)} \frac{1}{s(s+1)} \int_{-a}^{a} \varphi_{r}^{4} h^{s+1}\left(x, T_{1}\right) d x+A_{2} \int_{T_{1}}^{T_{2}} \int_{-r}^{r} h^{s+2} d x d t,
\end{aligned}
$$

where $A_{1}$ and $A_{2}$ depend on $s, \varphi_{1}, m, h_{0}$, and $T_{0}$ only. Taking $T_{1}=0$ and $T_{2}=T$ finishes the proof.

Given Lemma 2, the proof of Proposition 2 follows the methods of Bernis [3]. For completeness, we present the proof in Appendix C.

\subsection{Compactly supported weak solutions on the line.}

Theorem 2. (Nonnegative compactly supported weak solutions on the line) Let $h_{0} \geq 0, h_{0}$ compactly supported, and $h_{0} \in H^{1}(\mathbb{R})$. Let $m \geq 3$. The exponent $m$ and $\left\|h_{0}\right\|_{H^{1}(\mathbb{R})}$ determine a time of existence:

$$
T_{0}:=\frac{1}{4} \frac{1}{C_{\infty}(2 m-1)} \min \left\{1,\left\|h_{0}\right\|_{H^{1}(\mathbb{R})}^{1-2 m}\right\},
$$


where $C_{\infty}=C c_{\infty}^{2 m-1}$ and $C$ and $c_{\infty}$ are defined in Lemma 1 and its proof. They are independent of $\left\|h_{0}\right\|_{H^{1}}$.

Then there exists a nonnegative weak solution in the sense of distributions (5). Moreover, for all $-\frac{1}{2}<s<1$, there are constants $C$ and $D$ determined by $m, s, T_{0}$, and $\left\|h_{0}\right\|_{H^{1}}$ such that:

$$
\begin{aligned}
& \int_{0}^{T_{0}} \int_{-\infty}^{\infty}\left(h^{s / 2+1}\right)_{x x}^{2}(x, t) d x d t \leq C T_{0}+D \\
& \int_{0}^{T_{0}} \int_{-\infty}^{\infty}\left(h^{s / 4+1 / 2}\right)_{x}^{4}(x, t) d x d t \leq C T_{0}+D
\end{aligned}
$$

$$
\begin{aligned}
\left\|h\left(\cdot, T_{0}\right)\right\|_{H^{1}} & \leq F_{m}\left(T_{0},\left\|h_{0}\right\|_{H^{1}}\right)<\infty, \\
\left\|h\left(\cdot, T_{0}\right)\right\|_{H^{1}}^{2} & \leq\left\|h_{0}\right\|_{H^{1}}^{2} e^{C \int_{0}^{T_{0}}\|h(\cdot, s)\|_{L^{\infty}+\| h(\cdot, s)} \|_{L^{\infty}}^{2 m-1} d s},
\end{aligned}
$$

where

$$
\begin{aligned}
\mathcal{E}\left(T_{0}\right) & \leq \mathcal{E}(0), \\
\int_{0}^{T_{0}} \mathcal{E}(t) d t & \leq \mathcal{E}(0) T_{0},
\end{aligned}
$$

$$
\mathcal{E}(t):=\frac{1}{2} \int_{-\infty}^{\infty} h_{x}^{2}(x, t) d x-\frac{1}{m(m+1)} \int_{-\infty}^{\infty} h^{m+1}(x, t) d x .
$$

Furthermore, the solution satisfies the second moment inequality

$$
\int_{-\infty}^{\infty} x^{2} h\left(x, T_{0}\right) d x \leq \int_{-\infty}^{\infty} x^{2} h_{0}(x) d x+6 T_{0} \mathcal{E}(0) .
$$

Proof. Given the initial data $h_{0}$, definition (12) gives a time of existence for a nonnegative periodic weak solution

$$
\tilde{T}_{0}(a)=\frac{1}{2} \frac{1}{C_{a}(2 m-1)} \min \left\{1,\left\|h_{0}\right\|_{H^{1}(-a, a)}^{1-2 m}\right\} .
$$

This time depends on the $H^{1}(-a, a)$ norm of the initial data and on the interval size; we have made this dependence explicit above.

First, since $h_{0}$ is compactly supported, we choose the interval sufficiently large to contain its support: $\operatorname{supp}\left\{h_{0}\right\} \subset(-a, a)$. Then

$$
\left\|h_{0}\right\|_{H^{1}(-a, a)}=\left\|h_{0}\right\|_{H^{1}(\mathbb{R})} \Rightarrow \tilde{T}_{0}(a)=\frac{1}{2} \frac{1}{C_{a}(2 m-1)} \min \left\{1,\left\|h_{0}\right\|_{H^{1}(\mathbb{R})}^{1-2 m}\right\} .
$$


By definition, $C_{a}=C\left(c_{\infty}+1 / a\right)^{2 m-1}$, therefore $1 / C_{a}$ increases to $1 / C_{\infty}$ as $a \rightarrow$ $\infty$. This allows us to choose the interval larger still so that $1 / C_{a}>1 /\left(2 C_{\infty}\right)$. For such an interval, the periodic nonnegative weak solution exists up to time $T_{0}$, as desired, because $\tilde{T}_{0}(a)>T_{0}$.

Using the finite speed of propagation, we now choose the interval larger yet so that at time $T_{0}$ the periodic nonnegative weak solution has not reached ends of the interval. We can do this because from Proposition 2, there is a maximum speed of propagation $V$, where $V$ is determined by the initial data and $T_{0}$. Since both $V$ and $T_{0}$ are independent of the size of the interval $[-a, a]$, we can use them to choose the interval large enough: $\operatorname{diam}\left(\operatorname{supp}\left\{h_{0}\right\}\right)+V T_{0}<a-\delta$ for some $\delta>0$. From Theorem 1, there is a solution up to time $T_{0}$ and from Proposition 2 the solution is supported in $[-a+\delta, a-\delta]$ for all times $t \in\left[0, T_{0}\right]$. The solution $h$ satisfies the evolution equation in the sense of distributions (5) where the space integrals are over $[-a, a]$, rather than the line. However, since the solution is supported in $[-a+\delta, a-\delta]$ at all times, the periodic solution can be extended to be identically zero for $|x|>a$ and thus is a solution on the line. The bounds (41-44) follow immediately.

It remains to prove the second moment inequality (45). Let $\varphi$ be a smooth test function such that

$$
\varphi(x, t)= \begin{cases}x^{2} & x \in[-a+\delta, a-\delta] \\ 0 & x \notin\left[-a+\frac{\delta}{2}, a-\frac{\delta}{2}\right] .\end{cases}
$$

Using $\varphi$ in the definition of weak solution, and recalling that at all times the solution is supported in $[-a+\delta, a-\delta]$,

$$
\begin{aligned}
& \int_{-\infty}^{\infty} x^{2} h\left(x, T_{0}\right) d x-\int_{-\infty}^{\infty} x^{2} h_{0}(x) d x \\
& \quad= 3 \int_{0}^{T_{0}} \int_{-\infty}^{\infty} h_{x}^{2}(x, t) d x d t-\frac{2}{m+1} \int_{0}^{T_{0}} \int_{-\infty}^{\infty} h^{m+1}(x, t) d x d t \\
& \quad= 6 \int_{0}^{T_{0}} \mathcal{E}(t) d t-\frac{2(m-3)}{m(m+1)} \int_{0}^{T_{0}} \int_{-\infty}^{\infty} h^{m+1}(x, t) d x d t \\
& \quad \leq 6 \int_{0}^{T_{0}} \mathcal{E}(t) d t \leq 6 T_{0} \mathcal{E}(0),
\end{aligned}
$$

since $m \geq 3$. The last step used bound (44).

Up to this point in the article, we have considered the critical $(m=3)$ and supercritical $(m>3)$ cases of equation (2). We now turn to the subcritical case $(1 \leq m<3)$ and present the analogue of Theorem 2 . Its proof is very similar in spirit to the proof of Theorem 2, for this reason we give only a coarse sketch of its proof. Proposition 3 and Theorem 3 demonstrate that our blow-up conjecture is sharp for coefficients $f(y)=y$ and $g(y)=y^{m}$. 
Proposition 3. (Nonnegative compactly supported weak solutions on the line: the subcritical case) Let $h_{0} \geq 0, h_{0}$ compactly supported, $h_{0} \in H^{1}(\mathbb{R})$, and $1 \leq m<3$. Given $T_{0}<\infty$, there exists a nonnegative compactly supported weak solution in the sense of distributions (5). Moreover, for all $-\frac{1}{2}<s<1$, there are constants $C$ and $D$ determined by $m, s$, and $\left\|h_{0}\right\|_{H^{1}}$, and constants $c_{1}$ and $c_{2}$ determined by $m$ only, such that:

$$
\begin{aligned}
\int_{0}^{T_{0}} \int_{-\infty}^{\infty}\left(h^{s / 2+1}\right)_{x x}^{2}(x, t) d x d t \leq C T_{0}+D, \\
\int_{0}^{T_{0}} \int_{-\infty}^{\infty}\left(h^{s / 4+1 / 2}\right)_{x}^{4}(x, t) d x d t \leq C T_{0}+D, \\
\frac{1}{4}\left\|h\left(\cdot, T_{0}\right)\right\|_{H^{1}} \leq \mathcal{E}(0)+c_{2}\left\|h_{0}\right\|_{L^{1}}^{(m+3) /(3-m)}+c_{1}+\frac{1}{4}\left\|h_{0}\right\|_{L^{1}}^{2}, \\
\mathcal{E}\left(T_{0}\right) \leq \mathcal{E}(0), \\
\int_{0}^{T_{0}} \mathcal{E}(t) d t \leq \mathcal{E}(0) T_{0},
\end{aligned}
$$

where

$$
\mathcal{E}(t):=\frac{1}{2} \int_{-\infty}^{\infty} h_{x}^{2}(x, t) d x-\frac{1}{m(m+1)} \int_{-\infty}^{\infty} h^{m+1}(x, t) d x .
$$

Proof. Let $1 \leq m<3$. The first step is to find a priori bounds that are the analogue of Lemma 1. This is Proposition 2.2 in [13]:

$$
\frac{1}{4}|h(\cdot, t)|_{H^{1}}^{2} \leq \mathcal{E}\left(h_{0}\right)+c_{2}\left|h_{0}\right|_{L^{1}}^{(m+3) /(3-m)}+c_{1}+\frac{1}{4}\left|h_{0}\right|_{L^{1}}^{2}<\infty
$$

at all times $t$. The proof in [13] uses the mean of the solution, rather than the $L^{1}$ norm. These are the same since the function is nonnegative and the interval of unit length. The second step is to use the a priori bounds to prove the existence of smooth positive periodic approximate solutions, the analogue of Proposition 1. This is Proposition 3.2 in [13]. The third step is to prove that a subsequence of the approximate solutions converges to a nonnegative periodic weak solution, the analogue of Theorem 1. This is done in the proof of Theorem 3.4 of [13] for a different formulation the weak solution. However it is straightforward to modify the proof of Theorem 1 for the subcritical case. The fourth step is to prove finite speed of propagation for nonnnegative periodic weak solutions with compact initial data, the analogue of Proposition 2. The statement and proof of the subcritical analogue of this proposition and the necessary Lemma 2 are essentially unchanged; the only change is that the various constants do not depend on $T_{0}$. The final step is to go from nonnegative periodic weak solutions to nonnegative weak solutions on the line. This is done as in the proof of Theorem 2. 


\section{Finite-time Blow-UP OF THE COMPACTLY SUPPORTED WEAK SOLUTION FOR $m \geq 3$}

The results of Section 3 allow us to continue the solution in time as long as at each end-time the $H^{1}$ norm of the solution is finite. In this section, we use this fact, along with the second-moment inequality (45) to prove

Theorem 3. Let $h_{0}$ be nonnegative and compactly supported, $h_{0} \in H^{1}(\mathbb{R})$. If

$$
\mathcal{E}(0)=\frac{1}{2} \int_{-\infty}^{\infty} h_{0 x}^{2}(x) d x-\frac{1}{m(m+1)} \int_{-\infty}^{\infty} h_{0}^{m+1}(x) d x<0,
$$

then there is a singular time $T^{*}<\infty$ and a nonnegative weak solution in the sense of distributions (5) on $\left[0, T^{*}\right)$ such that

$$
\underset{t \uparrow T^{*}}{\limsup }\|h(\cdot, t)\|_{L^{\infty}(\mathbb{R})}=\limsup _{t \uparrow T^{*}}\|h(\cdot, t)\|_{H^{1}(\mathbb{R})}=\infty .
$$

In this theorem, we prove that the $H^{1}$ and $L^{\infty}$ norms blow up, although we do not know that the $H^{1}$ norm is continuous in time. However, the solution is $C^{1 / 2,1 / 8}$ and in particular the $L^{\infty}$ norm is continuous in time up to the blow-up time, and the $L^{\infty}$ and $H^{1}$ norms are tightly coupled through inequalities (8) and (11). Also, we note that $M(t)=\sup _{0<s<t}\|h(\cdot, s)\|_{L^{\infty}}$ is an increasing function of $t$ and $\lim M(t)=\infty$ as $t \rightarrow T^{*}$.

Proof. First, we construct a sequence of times $T_{0}<T_{1}<\cdots$ and extend the weak solution $h$ from the time interval $\left[0, T_{i}\right]$ to the time interval $\left[0, T_{i+1}\right]$.

By Theorem 2, there is a weak solution $h$ on a time interval $\left[0, T_{0}\right]$. At time $T_{0}$, the solution is compactly supported and has finite $H^{1}$ norm (bounds (41-42)). Taking it as an initial datum, its $H^{1}$ norm determines a time interval of existence

$$
T_{1}-T_{0}=\frac{1}{4} \frac{1}{C_{\infty}(2 m-1)} \min \left\{1,\left\|h\left(\cdot, T_{0}\right)\right\|_{H^{1}(\mathbb{R})}^{1-2 m}\right\},
$$

by (40). Applying Theorem 2 to the time interval $\left[T_{0}, T_{1}\right]$, we have a weak solution that is compactly supported at all times and that satisfies bounds (41-44), with the time interval $\left[0, T_{0}\right]$ replaced by $\left[0, T_{1}\right]$.

Thus, applying the second-moment inequality (45) twice,

$$
\begin{aligned}
\int_{-\infty}^{\infty} x^{2} h\left(x, T_{1}\right) d x & \leq \int_{-\infty}^{\infty} x^{2} h\left(x, T_{0}\right) d x+6\left(T_{1}-T_{0}\right) \mathcal{E}\left(T_{0}\right) \\
& \leq \int_{-\infty}^{\infty} x^{2} h_{0}(x) d x+6\left(T_{1}-T_{0}\right) \mathcal{E}\left(T_{0}\right)+6 T_{0} \mathcal{E}(0) \\
& \leq \int_{-\infty}^{\infty} x^{2} h_{0}(x) d x+6 T_{1} \mathcal{E}(0)
\end{aligned}
$$


The last step used bound (43): $\mathcal{E}\left(T_{0}\right) \leq \mathcal{E}(0)$.

Continuing the construction inductively, we have a sequence of times $T_{0}<$ $T_{1}<\cdots<T_{n}<\cdots$ and a nonnegative weak solution in the sense of distributions (5) on the time interval $\left[0, T^{*}\right)$, where

$$
T^{*}=\lim _{n \rightarrow \infty} T_{n}
$$

At each time $T_{n}$, the $H^{1}$ norm of $h$ is finite, satisfying bounds (41-42). Also, at each time,

$$
\int_{-\infty}^{\infty} x^{2} h\left(x, T_{n}\right) d x \leq \int_{-\infty}^{\infty} x^{2} h_{0}(x) d x+6 T_{n} \mathcal{E}(0) .
$$

Since $\mathcal{E}(0)<0$, if $T_{n} \rightarrow \infty$, then for large times the right-hand side would be negative: an impossibility. Therefore $\lim _{n \rightarrow \infty} T_{n}=T^{*}<\infty$. This implies that

$$
\frac{1}{4} \frac{1}{C_{\infty}(2 m-1)} \min \left\{1,\left\|h\left(\cdot, T_{n}\right)\right\|_{H^{1}(\mathbb{R})}^{1-2 m}\right\}=T_{n+1}-T_{n} \rightarrow 0 .
$$

That is, the $H^{1}$ norms at times $T_{n}$ must blow up. It then follows from bound (42) that the $L^{\infty}$ norm must also blow up:

$$
\underset{t \uparrow T^{*}}{\limsup }\|h(\cdot, t)\|_{L^{\infty}}=\limsup _{t \uparrow T^{*}}\|h(\cdot, t)\|_{H^{1}}=\infty .
$$

We close by presenting simple initial data for which $\mathcal{E}(0)<0$. Consider

$$
h_{0}(x)= \begin{cases}\lambda(1+\cos (\lambda x)) & \text { for }-\frac{\pi}{\lambda} \leq x \leq \frac{\pi}{\lambda}, \\ 0 & \text { otherwise. }\end{cases}
$$

For all values of $\lambda, h_{0}$ has mean value $2 \pi$,

$$
\frac{1}{2} \int_{-\pi / 2}^{\pi / 2} h_{0 x}^{2}(x) d x=\frac{\lambda^{3} \pi}{2}, \quad \text { and } \quad \frac{1}{m(m+1)} \int_{-\pi / 2}^{\pi / 2} h_{0}^{m+1}(x) d x \sim \lambda^{m} .
$$

It follows immediately that for $m>3, \mathcal{E}(0)<0$ for $\lambda$ sufficiently large. For example,

$$
\begin{array}{ll}
\text { for } m=3 \mathcal{E}(0)=-\frac{11}{48} \pi \lambda^{3}<0 & \text { if } \lambda>0, \\
\text { for } m=4 \mathcal{E}(0)=\frac{1}{2} \pi \lambda^{3}-\frac{63}{80} \pi \lambda^{4}<0 & \text { if } \lambda>\frac{40}{63} .
\end{array}
$$




\section{CONCLUSIONS}

In this paper we prove the existence of a particular weak solution of

$$
h_{t}=-\left(h^{n} h_{x x x}\right)_{x}-\left(h^{m} h_{x}\right)_{x},
$$

with $m \geq 3$ and $n=1$. The solution is nonnegative, is compactly supported, and has a finite-time singularity of the form $\lim \sup \|h(\cdot, t)\|_{L^{\infty}}=\infty$ and $\limsup \|h(\cdot, t)\|_{H^{1}}=\infty$ as $t \rightarrow T^{*}$. In [13], we proved that solutions are globally bounded whenever $m<n+2$. Thus, the results in this paper prove our conjecture that singularities can occur only when $m \geq n+2$ for the specific case $n=1$.

To be precise, in [13] we considered the initial value problem on a periodic domain, while in this paper we consider the initial value problem on the line. However, in both cases compactly supported initial data are shown to yield compactly supported solutions. Hence the two initial value problems coincide for short times or large intervals in space.

The finite-time singularity proof relies on an identity involving the second moment of the solution, $\int x^{2} h d x$. This identity implies that certain initial data cannot yield solutions that exist past a time $T^{*}$. A separate argument then implies that the lim-sup of the $L^{\infty}$ and $H^{1}$ norms must become infinite in finite time. While our blow-up conjecture is stated for general equations of the form

$$
h_{t}=-\left(f(h) h_{x x x}\right)_{x}-\left(g(h) h_{x}\right)_{x},
$$

the second-moment identity is special to the coefficient $f(y)=y$ and does not immediately generalize to other coefficients. In fact, we used a second-moment inequality that followed from the identity. It may be possible to prove such an inequality directly for other supercritical pairs of coefficients $f$ and $g$. Numerical simulations suggest that the blow-up conjecture is sharp for other coefficients $f$ and $g$.

For $f(y)=y^{n}$ and $g(y)=y^{m}$, the conjecture refers to $m=n+2$ as the critical case and to $m>n+2$ as the supercritical case. For $n=1$, the finite-time singularity proof states that if the initial data initially has negative energy

$$
\frac{1}{2} \int h_{x}^{2}(x) d x-\frac{1}{m(m+1)} \int h^{m+1}(x) d x<0,
$$

then it yields a solution that becomes singular in finite time. For the critical $m=3$ case, this requires that the initial mass of the solution be greater than some value $M_{c}$ (as proved in [13]). However, for the supercritical $m>3$ case one can find initial data with arbitrarily small mass that has negative energy.

Numerical simulations [8] show that, for the critical case, there is a continuous family of linearly stable similarity solutions describing blow-up. The blow-up profile appears to be determined by the mass of the initial data. This is to be contrasted with the supercritical case, where simulations find a discrete family of 
similarity solutions only one of which is linearly stable. Simulations of the initial value problem find that this profile is universally selected. That solution shows a blow-up with zero mass.

Finally, we note that the scaling arguments that led to the blow-up conjecture for the 1-d equation (1) can be applied [13] to the 2-d equation

$$
h_{t}=-\nabla \cdot(f(h) \nabla \Delta h)-\nabla \cdot(g(h) \nabla h) .
$$

For $f(y)=y^{n}$ and $g(y)=y^{m}$, the conjecture is that if $m<n+1$, then finite-time blow-up is impossible, while if $m \geq n+1$, then finite-time blow-up is possible. The existence theory for the 2 -d equation with $g \equiv 0$ has been welldeveloped $[36,37]$, however the 2 -d equation with $g \neq 0$ has yet to be addressed.

APPENDIX A. $L^{2}$ BOUND OF THE FLUX

The evolution equation is of the form

$$
h_{\varepsilon t}+\left(f_{\varepsilon}\left(h_{\varepsilon}\right) h_{\varepsilon x x x}+g_{\varepsilon}\left(h_{\varepsilon}\right) h_{\varepsilon x}\right)_{x}=0 .
$$

We now prove that the flux is a priori bounded in $L^{2}\left(Q_{T_{0}}\right)$ with a bound independent of $\varepsilon$ :

$$
\int_{0}^{T_{0}} \int_{-a}^{a}\left|f_{\varepsilon}\left(h_{\varepsilon}(x, t)\right) h_{\varepsilon x x x}(x, t)+g_{\varepsilon}\left(h_{\varepsilon}(x, t)\right) h_{\varepsilon x}(x, t)\right|^{2} d x d t \leq A .
$$

The upper bound $A$ depends on $\left\|h_{0}\right\|_{H^{1}}$, on the interval size $a$, and on the exponent $m$ :

$$
\begin{aligned}
& \int_{0}^{T_{0}} \int_{-a}^{a}\left|f_{\varepsilon}\left(h_{\varepsilon}(x, t)\right) h_{\varepsilon x x x}(x, t)+g_{\varepsilon}\left(h_{\varepsilon}(x, t)\right) h_{\varepsilon x}(x, t)\right|^{2} d x d t \\
& \leq\left\|f_{\varepsilon}\left(h_{\varepsilon}\right)\right\|_{L^{\infty}\left(Q_{T_{0}}\right) \int_{0}^{T_{0}} \int_{-a}^{a} f_{\varepsilon}\left(h_{\varepsilon}(x, t)\right)\left[h_{\varepsilon x x x}(x, t)\right.} \\
& \left.\quad+\frac{g_{\varepsilon}\left(h_{\varepsilon}(x, t)\right)}{f_{\varepsilon}\left(h_{\varepsilon}(x, t)\right)} h_{\varepsilon x}(x, t)\right]^{2} d x d t \\
& \quad \leq\left\|h_{\varepsilon}\right\|_{L^{\infty}\left(Q_{T_{0}}\right) \int_{0}^{T_{0}} \int_{-a}^{a} f_{\varepsilon}\left(h_{\varepsilon}(x, t)\right)\left[h_{\varepsilon x x x}(x, t)\right.} \\
& \left.\quad+\frac{g_{\varepsilon}\left(h_{\varepsilon}(x, t)\right)}{f_{\varepsilon}\left(h_{\varepsilon}(x, t)\right)} h_{\varepsilon x}(x, t)\right]^{2} d x d t \\
& \leq C \int_{0}^{T_{0}} \int_{-a}^{a} f_{\varepsilon}\left(h_{\varepsilon}(x, t)\right)\left[h_{\varepsilon x x x}(x, t)\right. \\
& \left.\quad+\frac{g_{\varepsilon}\left(h_{\varepsilon}(x, t)\right)}{f_{\varepsilon}\left(h_{\varepsilon}(x, t)\right)} h_{\varepsilon x}(x, t)\right]^{2} d x d t
\end{aligned}
$$


At step (46) we used the bound

$$
f_{\varepsilon}(y)=\frac{y^{5}}{\varepsilon y+y^{4}} \leq y .
$$

At step (47) we used the energy identity (24)

$$
\begin{aligned}
\int_{0}^{T_{0}} \int_{-a}^{a} f_{\varepsilon}\left(h_{\varepsilon}(x, t)\right)\left[h_{\varepsilon x x x}(x, t)+\frac{g_{\varepsilon}\left(h_{\varepsilon}(x, t)\right)}{f_{\varepsilon}\left(h_{\varepsilon}(x, t)\right)} h_{\varepsilon x}(x, t)\right]^{2} d x d t \\
\leq \mathcal{F}_{\varepsilon}(0)-\mathcal{F}_{\varepsilon}\left(T_{0}\right) .
\end{aligned}
$$

Recall that

$$
\mathcal{E}_{\varepsilon}\left(T_{0}\right)=\frac{1}{2} \int_{-a}^{a} h_{\varepsilon_{x}}^{2}\left(x, T_{0}\right) d x-\int_{-a}^{a} G_{\varepsilon}\left(h_{\varepsilon}\left(x, T_{0}\right)\right) d x .
$$

The first integral is bounded using the $H^{1}$ control of Lemma 1, and the second integral is bounded by using (20) at time $t=T_{0}$. Combining these upper bounds, we find the desired constant $A$.

\section{APPENDiX B. UNIFORM CONVERGENCE OF $f_{\varepsilon}^{\prime \prime}(y) y^{7 / 4}$ TO ZERO}

Lemma 3. For

$$
f_{\varepsilon}(y)=\frac{y^{5}}{\varepsilon y+y^{4}},
$$

there is a constant $C$ such that

$$
y \geq 0 \Rightarrow\left|f_{\varepsilon}^{\prime \prime}(y) y^{7 / 4}\right| \leq C \varepsilon^{1 / 4} .
$$

Proof. We define

$$
F_{\varepsilon}(y):=f_{\varepsilon}^{\prime \prime}(y) y^{7 / 4}=6 y^{15 / 4} \frac{2 \varepsilon-y^{3}}{\left(\varepsilon+y^{3}\right)^{3}} .
$$

Its first derivative is:

$$
F_{\varepsilon}^{\prime}(y)=\frac{9}{2} \varepsilon y^{11 / 4} \frac{10 \varepsilon^{2}-23 \varepsilon y^{3}+3 y^{6}}{\left(\varepsilon+y^{3}\right)^{4}} .
$$

$F_{\varepsilon}$ has two critical points in $(0, \infty)$ :

$$
y_{ \pm}^{3}=\varepsilon \frac{23 \pm \sqrt{409}}{6} .
$$


Evaluating $F_{\varepsilon}^{\prime \prime}$ at the critical points, we find $y_{-}$is a local maximum and $y_{+}$is a local minimum. For fixed $\varepsilon, F_{\varepsilon}(y)$ goes to zero as $y \rightarrow \infty$, is positive on $[0, \sqrt[3]{2 \varepsilon})$, and is negative on $(\sqrt[3]{2 \varepsilon}, \infty)$, therefore $y_{ \pm}$are global minima and maxima. Evaluating at $h_{\varepsilon}$ at $y_{ \pm}$we find

$$
\max _{y \in[0, \infty)}\left|F_{\varepsilon}(y)\right| \leq C \varepsilon^{1 / 4} .
$$

\section{Appendix C. Proof of Finite SPEED of PROpagation}

For completeness, we present the proof of finite speed of propagation. What follows is, in essence, Bernis's argument for the equation $h_{t}=-\left(h^{n} h_{x x x}\right)_{x}$ with $0<n<2[3]$.

Proof of Proposition 2. We prove finite speed of propagation in two steps. We first prove finite speed of propagation for short times. The methods in this first part will apply to any initially dry region $\omega$, proving finite speed of propagation as defined in Section 3.3. However, we only present the argument for the dry regions $\left(-a,-a_{0}\right)$ and $\left(a_{0}, a\right)$ where the initial data is supported in $\left(-a_{0}, a_{0}\right)$ and the solution is periodic on the interval $(-a, a)$. We then prove that, by taking a sufficiently large domain $(-a, a)$, the short-time result for the dry regions $\left(-a,-a_{0}\right)$ and $\left(a_{0}, a\right)$ holds up to the time $T_{0}$ of the a priori $H^{1}$ bound of Lemma 1. This then gives the desired control on the diameter of the support of the weak solution.

First stage. Given compactly supported initial data $h_{0}$, find $a_{0}$ so that $\operatorname{supp}\left\{h_{0}\right\} \subset\left(-a_{0}, a_{0}\right)$. For $a$ such that $a_{0}<a$, we prove there is a short time $T^{*}>0$ such that $-a<-3 a / 4-a_{0} / 4<\zeta_{L}(t)$ and $\zeta_{R}(t)<a_{0} / 4+3 a / 4<a$ for all $t \leq T^{*}(a)$. We present the proof for the right interface $\zeta_{R}(t)$. The proofs for the left interface and for other dry regions $\omega$ are essentially identical.

Define $x_{m}=\left(a_{0}+a\right) / 2$ and translate $x_{m}$ to the origin, making $h_{0} \equiv 0$ on $\left(-r_{0}, r_{0}\right)$, where $r_{0}=\left(a-a_{0}\right) / 2$. Take

$$
\begin{aligned}
& \varphi_{1}(x)=\left(1-x^{2}\right)_{+}, \\
& \varphi_{r}(x)=\frac{1}{r}\left(r^{2}-x^{2}\right)_{+}, \quad \text { for all } r \leq r_{0} .
\end{aligned}
$$

Lemma 2 applies for such test functions, hence for all $T \leq T_{0}$

$$
\begin{aligned}
& \frac{1}{s(s+1)} \int_{-r}^{r} \frac{1}{r^{4}}\left(r^{2}-x^{2}\right)^{4} h^{s+1}(x, T) d x \\
& \quad+A_{1} \int_{0}^{T} \int_{-r}^{r} \frac{1}{r^{4}}\left(r^{2}-x^{2}\right)^{4}\left(h^{(s+2) / 2}\right)_{x x}^{2}(x, t) d x d t \\
& \quad \leq A_{2} \int_{0}^{T} \int_{-r}^{r} h^{s+2}(x, t) d x d t .
\end{aligned}
$$


Since

$$
(r-|x|)_{+} \leq \varphi_{r}(x) \leq 2(r-|x|)_{+},
$$

this becomes

$$
\begin{array}{r}
\int_{-r}^{r}(r-|x|)^{4} h(x, T)^{s+1} d x+A_{1} \int_{0}^{T} \int_{-r}^{r}(r-|x|)^{4}\left(h^{(s+2) / 2}(x, t)\right)_{x x}^{2} d x d t \\
\leq A_{2} \int_{0}^{T} \int_{-r}^{r} h^{s+2}(x, t) d x d t
\end{array}
$$

Introducing

$$
w=h^{(s+2) / 2}, \quad q=2-\frac{2}{s+2} \in\left(\frac{2}{3}, \frac{4}{3}\right),
$$

the inequality is

$$
\begin{array}{r}
\int_{-r}^{r}(r-|x|)^{4} w^{q}(x, T) d x+A_{1} \int_{0}^{T} \int_{-r}^{r}(r-|x|)^{4}(x) w_{x x}^{2}(x, t) d x d t \\
\leq A_{2} \int_{0}^{T} \int_{-r}^{r} w^{2}(x, t) d x d t .
\end{array}
$$

Taking the supremum over time, for any $T \leq T_{0}$ we have

$$
\begin{array}{r}
\sup _{0 \leq t \leq T} \int_{-r}^{r}(r-|x|)^{4} w^{q}(x, t) d x+A_{1} \int_{0}^{T} \int_{-r}^{r}(r-|x|)^{4}(x) w_{x x}^{2}(x, t) d x d t \\
\leq A_{2} \int_{0}^{T} \int_{-r}^{r} w^{2}(x, t) d x d t
\end{array}
$$

We introduce the notation

$$
\begin{aligned}
E_{S}(r, T) & :=\int_{0}^{T} \int_{-r}^{r}(r-|x|)^{s} w_{x x}^{2}(x, t) d x d t, \quad \text { and } \\
F(r, T) & :=\sup _{0 \leq t \leq T} \int_{-r}^{r}(r-|x|)^{4} w^{q}(x, t) d x .
\end{aligned}
$$

Hence the inequality is

$$
F(r, T)+A_{1} E_{4}(r, T) \leq A_{2} \int_{0}^{T} \int_{-r}^{r} w^{2}(x, t) d x d t .
$$

Bernis [3] proves the following interpolation inequality: 
Lemma. Let $0<q<2$ and $0<r<\infty$. Then there exists a constant $K_{1}$ depending only on $q$ such that

$$
\begin{aligned}
& K_{1} \int_{-r}^{r} w^{2}(x, t) d x \\
& \leq\left(\int_{-r}^{r} w_{x x}^{2}(x, t) d x\right)^{d}\left(\int_{-r}^{r}(r-|x|)^{4}|w(x, t)|^{q} d x\right)^{(2(1-d)) / q} \\
& \quad+r^{-2 v}\left(\int_{-r}^{r}(r-|x|)^{4}|w(x, t)|^{q} d x\right)^{2 / q},
\end{aligned}
$$

where

$$
d=\frac{10-q}{10+3 q} \quad \text { and } \quad v=\frac{10-q}{2 q} .
$$

In fact, one can prove this interpolation inequality for any moment on the right-hand side of (49). The fourth moment is chosen so that the exponents worked cleanly in the proof of Lemma 2.

Integrating inequality (49) in time, and using the Hölder inequality with $p=$ $1 / d$, we find

$$
\int_{0}^{T} \int_{-r}^{r} w^{2}(x, t) d x d t \leq T^{1-d} E_{0}^{d}(r, T) F^{(2(1-d)) / q}(r, T)+\operatorname{Tr}^{-2 v} F^{2 / q}(r, T) .
$$

Therefore for any $T \leq T_{0}$ inequality (48) becomes

$$
\begin{aligned}
F(r, T)+A_{1} E_{4}(r, T) \leq A_{2} T^{1-d} E_{0}^{d}(r, T) F^{(2(1-d)) / q}(r, T) & \\
& +A_{2} T r^{-2 v} F^{2 / q}(r, T)
\end{aligned}
$$

For any $r \leq r_{0}$ and $T \leq T_{0}$,

$F(r, T) \leq F\left(r_{0}, T_{0}\right) \Rightarrow F^{2 / q}(r, T) \leq F(r, T) F^{2 / q-1}\left(r_{0}, T_{0}\right)=: M\left(r_{0}, T_{0}\right) F(r, T)$.

Choose $T^{*}$ such that

$$
A_{2} T^{*} M\left(r_{0}, T_{0}\right)\left(\frac{r_{0}}{2}\right)^{-2 v} \leq \frac{1}{2}
$$

Then for $r_{0} / 2 \leq r \leq r_{0}$ and $T \leq T^{*}$, we have

$$
A_{2} \operatorname{Tr}^{-2 v} F^{2 / q}(r, T) \leq \frac{F}{2} .
$$


The lower bound on $r$ is needed since the exponent $-2 v$ in (51) is negative. Thus for $T \leq T^{*}$ and $r_{0} / 2 \leq r \leq r_{0}$, inequality (50) becomes

$$
F(r, T)+A_{1} E_{4}(r, T) \leq A_{2} T^{1-d} E_{0}^{d}(r, T) F^{(2(1-d)) / q}(r, T)+\frac{1}{2} F(r, T) .
$$

Applying Young's inequality with $p=q /(2(1-d))$ to the first term on the righthand side, for $T \leq T_{0}$ and $r_{0} / 2 \leq r \leq r_{0}$,

$$
E_{4}(r, T) \leq K^{\eta} E_{0}^{9}(r, T), \quad \text { where } K^{\eta}:=C T^{\eta} r_{0}^{\mu}
$$

and

$$
\eta=\frac{q(1-d)}{q-2(1-d)}=4 \frac{s+1}{4 s+5}, \quad 9=\frac{q d}{q-2(1-d)}=\frac{4 s+9}{4 s+5} .
$$

The constant $C$ depends on $s, \varphi_{1}, m, h_{0}$, and $T_{0}$. Inequality (52) is a differential inequality because $\left(d^{4} / d r^{4}\right) E_{4}(r, T)=4 ! E_{0}(r, T)$. For such differential inequalities, Bernis [3] proved:

Lemma. Assume the differential inequality (52) holds for $r_{0} / 2 \leq r \leq r_{0}$. Define

$$
r_{1}(T)=r_{0}-\frac{\vartheta+3}{9-1} K^{\eta / 4}\left(E_{0}\left(r_{0}, T\right)\right)^{(\vartheta-1) / 4} .
$$

If $r_{0} / 2 \leq r_{1}(T) \leq r_{0}$, then $E_{0}(r, T)=0$ for all $r \in\left[0, r_{1}(T)\right]$. that is

To apply the lemma, we need to ensure that $r_{1}(T) \geq r_{0} / 2$ for all $T \leq T^{*}$,

$$
\frac{\vartheta+3}{9-1} C T^{\eta / 4}\left(E_{0}\left(r_{0}, T\right)\right)^{(\vartheta-1) / 4} \leq \frac{r_{0}}{2} .
$$

The length $r_{0}$ is fixed. The function $T \rightarrow E_{0}\left(r_{0}, T\right)$ is nondecreasing with $E_{0}\left(r_{0}, 0\right)$ $=0$, and both exponents $\eta / 4$ and $(\vartheta-1) / 4$ are positive. As a result, the inequality holds, choosing $T^{*}$ smaller if need be. By the same logic, $r_{1}\left(T^{*}\right) \leq r_{1}(T)$ for $T \leq T^{*}$. It follows that $E_{0}(r, T)=0$ for all $r \leq r_{1}\left(T^{*}\right)$ and $T \leq T^{*}$. Translating back to the original coordinates, this implies $\zeta_{R}(T) \leq\left(3 a_{0}+a\right) / 4<a$ for all $T \leq T^{*}$, as desired. A similar argument proves $-a<\zeta_{L}(T)$ for all $T \leq T^{*}$.

Second Stage.

We now prove that, by taking the interval $(-a, a)$ sufficiently large, $-a<$ $\zeta_{L}(t)$ and $\zeta_{R}(t)<a$ for all $t \leq T_{0}$, where $T_{0}$ is the time from the a priori $H^{1}$ bound of Lemma 1.

Again, we present the argument for the right interface $\zeta_{R}(t)$. The argument for the left interface is essentially identical. Recall that the initial data was supported on $\left(-a_{0}, a_{0}\right), x_{m}=\left(a+a_{0}\right) / 2, r_{0}=\left(a-a_{0}\right) / 2$, and $x_{m}$ has been translated to the origin. Following Bernis, we define

$$
\hat{T}=\sup \{t \in(0, \infty) \mid \exists \delta=: \delta(t) \text { such that } h \equiv 0 \text { on }(-\delta, \delta) \times(0, t)\} .
$$


From the first stage, $T^{*} \leq \hat{T}$. We now prove that by choosing $a$ sufficiently large, $\hat{T} \geq T_{0}$.

Fix $T \leq \hat{T}$. By definition, $\zeta_{R}(T) \leq-\delta(T)<0$. We use $\delta(T)$ to define a test function $\varphi_{r}$. Take $\varphi_{1}(x)=(x+1)_{+} \vartheta(x)$, where $\vartheta \in C^{\infty}(\mathbb{R})$ and

$$
\mathcal{\vartheta}= \begin{cases}1 & \text { for } x \leq 0 \\ 0 & \text { for } x \geq \frac{\delta(T)}{r_{0}} .\end{cases}
$$

Recalling $\varphi_{r}(x)=r \varphi_{1}(x / r)$, by construction $\varphi_{r}(x)=(x+r)$ on $(-r, 0)$. Also, since $r \leq r_{0}$, the support of $\varphi_{1}$ is contained in $(-r, \delta(T))$.

Taking $\varphi_{r}$ in (27), we have

$$
\begin{array}{r}
\frac{1}{s(s+1)} \int_{-r}^{0} \varphi_{r}^{4} h^{s+1}(x, T) d x+A_{1} \int_{0}^{T} \int_{-r}^{0} \varphi_{r}^{4}\left(h^{(s+2) / 2}\right)_{x x}^{2}(x, t) d x d t \\
\leq A_{2} \int_{0}^{T} \int_{-r}^{r} h^{s+2}(x, t) d x d t .
\end{array}
$$

Here, we used the fact that by construction $h(x, t)=0$ for all $x \in(0, r), t \in$ $(0, T)$. Again, we introduce $w$ and $q$, yielding

$$
\begin{aligned}
\frac{1}{s(s+1)} \int_{-r}^{0} \varphi_{r}^{4} w^{q}(x, T) d x+A_{1} \int_{0}^{T} \int_{-r}^{0} \varphi_{r}^{4} w_{x x}^{2} d x d t & \\
& \leq A_{2} \int_{0}^{T} \int_{-r}^{r} w^{2}(x, t) d x d t
\end{aligned}
$$

For any time $t \leq T$, the inequality (53) holds since $w \equiv 0$ on $(0, r) \times(0, T)$. Since $\varphi_{r}(x)=(x+r)$ on $(-r, 0)$, we have

$$
\begin{aligned}
\frac{1}{s(s+1)} \int_{-r}^{0}(x+r)^{4} w^{q}(x, t) d x+A_{1} \int_{0}^{t} \int_{-r}^{0}(x+r)^{4} w_{x x}^{2}(x, t) d x d t \\
\leq A_{2} \int_{0}^{t} \int_{-r}^{0} w^{2}(x, t) d x d t
\end{aligned}
$$

Taking the supremum over $t$,

$$
\begin{array}{r}
\sup _{0 \leq t \leq T} \frac{1}{s(s+1)} \int_{-r}^{0}(x+r)^{4} w^{q}(x, t) d x+A_{1} \int_{0}^{T} \int_{-r}^{0}(x+r)^{4} w_{x x}^{2}(x, t) d x d t \\
\leq A_{2} \int_{0}^{T} \int_{-r}^{0} w^{2}(x, t) d x d t
\end{array}
$$


Let $z=-r$ and introduce

$$
\hat{F}(z, T)=\sup _{0 \leq t \leq T} \int_{z}^{0}(x-z)^{4} w^{q}(x, t) d x,
$$

and

$$
\hat{E}_{S}(z, T)=\int_{0}^{T} \int_{z}^{0}(x-z)^{s} w_{x x}^{2}(x, t) d x d t
$$

Then

$$
\hat{F}(z, T)+A_{1} \hat{E}_{4}(z, T) \leq A_{2} \int_{0}^{T} \int_{z}^{0} w^{2}(x, t) d x d t .
$$

Bernis [3] proves the following interpolation inequality:

Lemma. Let $0<q<2$ and $-\infty<z<b \leq \infty$. Assume that either $b=\infty$ or $v(b)=v^{\prime}(b)=0$. Then

$$
K_{2} \int_{z}^{b} v^{2}(x) d x \leq\left(\int_{z}^{b} v_{x x}^{2}(x) d x\right)^{d}\left(\int_{z}^{b}(x-z)^{4} v^{q}(x) d x\right)^{2(1-d) / q}
$$

$K_{2}$ depends on a only.

By construction, $w \equiv 0$ in a neighborhood of $x=0$ for all times $0 \leq t \leq T$, hence the lemma applies with $b=0$. This leads to

$$
K_{2} \int_{0}^{T} \int_{z}^{0} w^{2}(x, t) d x d t \leq \hat{F}(z, T)^{2(1-d) / q} T^{1-d} \hat{E}_{0}(z, T)^{d},
$$

hence

$$
\hat{F}(z, T)+A_{1} \hat{E}_{4}(z, T) \leq A_{3} \hat{F}(z, T)^{2(1-d) / q} T^{1-d} \hat{E}_{0}(z, T)^{d},
$$

where $A_{3}$ depends on $s, \varphi_{1}, m, h_{0}$, and $T_{0}$ only. Again, we use Young's inequality with $p=q /(2(1-d))$ to cancel $\hat{F}(z, T)$ from the left-hand side, yielding

$$
\hat{E}_{4}(z, T) \leq C T^{\eta} \hat{E}_{0}(z, T)^{\vartheta}=K^{\eta} \hat{E}_{0}(z, T)^{\vartheta}, \quad \eta=\frac{4 q}{2+3 q}, \quad \vartheta=\frac{10-q}{2+3 q} .
$$

We note that $\eta>0$ and $\vartheta>1$. For such differential inequalities, Bernis [3] proved:

Lemma. Let $K>0, \eta \in \mathbb{R}, \vartheta>1$, and

$$
\hat{E}_{4}(z, T) \leq C K^{\eta} \hat{E}_{0}(z, T)^{\vartheta}
$$


for $z_{0} \leq z \leq 0$. Define

$$
z_{1}(T)=z_{0}+\frac{\vartheta+3}{\vartheta-1} K^{\eta / 4} \hat{E}_{0}\left(z_{0}, T\right)^{(\vartheta-1) / 4} .
$$

If $z_{1}(T)<0$, then $\hat{E}_{0}(z, T) \equiv 0$ on $\left[z_{1}(T), 0\right)$.

Now

$$
\begin{aligned}
z_{1}(T) & =z_{0}+\frac{\vartheta+3}{\vartheta-1} C T^{\eta / 4} \hat{E}_{0}\left(z_{0}, T\right)^{(\vartheta-1) / 4} \\
& \leq z_{0}+\frac{\vartheta+3}{\vartheta-1} C T_{0}^{\eta / 4} \hat{E}_{0}\left(z_{0}, T_{0}\right)^{(\vartheta-1) / 4} \\
& \leq z_{0}+\frac{\vartheta+3}{\vartheta-1} C T_{0}^{\eta / 4}\left(\int_{0}^{T_{0}} \int_{-a}^{a} w_{x x}^{2}(x, t) d x d t\right)^{(\vartheta-1) / 4} \\
& \leq z_{0}+\frac{\vartheta+3}{\vartheta-1} C T_{0}^{\eta / 4} D\left(s, m, h_{0}, T_{0}\right)^{(\vartheta-1) / 4} .
\end{aligned}
$$

We want $z_{1}(T) \leq 0$; this will be true for all $0 \leq T \leq t\left(z_{0}\right)$ where

$$
t\left(z_{0}\right)^{\eta / 4}=\frac{\vartheta-1}{\vartheta+3} \frac{-z_{0}}{C D\left(s, m, h_{0}, T_{0}\right)^{(\vartheta-1) / 4}} .
$$

In the last step, we used the bound inherited from (13) with $s=0$.

Since $\eta>0$, this shows that as $z_{0} \rightarrow 0, t\left(z_{0}\right) \rightarrow 0$, as expected. To ensure $t\left(z_{0}\right) \geq T_{0}$, we take $z_{0}$ sufficiently negative. Since $z_{0}=-r_{0}=-\left(a-a_{0}\right) / 2$, this corresponds to taking $a$ sufficiently large. This then guarantees

$$
\zeta_{r}(T) \leq z_{1}(t)+\frac{a+a_{0}}{2} \leq \frac{a+a_{0}}{2} \leq a
$$

for all $T \leq T_{0}$, finishing the proof.

\section{ACKNOWLEDGMents}

We thank Andrew J. Bernoff for pointing out the formal second moment argument upon which the finite-time blow-up proof hinges. M. P. thanks Richard S. Laugesen for useful mathematical conversations.

A. B. was supported by an ONR Young Investigator/PECASE award and an Alfred P. Sloan Research Fellowship. M. P. was supported by NSF grant number DMS-9971392 and an Alfred P. Sloan Research Fellowship.

\section{REFERENCES}

[1] E. BeretTA, M. Bertsch \& R. DAL PASSO, Nonnegative solutions of a fourth order nonlinear degenerate parabolic equation, Arch Ration Mech An 129 (1995), 175-200.

[2] F. BERNIS, Change of sign of the solutions to some parabolic problems, In: Nonlinear Analysis and Applications (Arlington, Tex., 1986), Dekker, New York, 1987 pp. 75-82. 
[3] - Finite speed of propagation and continuity of the interface for thin viscous flows, Adv. Differential Equations 1 (1996), 337-368.

[4] - Finite speed of propagation for thin viscous flows when $2 \leq n<3$, CR Acad Sci Paris Sér I-Math 322 (1996), 1169-1174.

[5] F. BERNIS \& A. FRIEDMAN, Higher order nonlinear degenerate parabolic equations, J Differ Equations 83 (1990), 179-206.

[6] A. J. BERNOFF, personal communication, 1998.

[7] A. J. BERNOFF \& A. L. BERTOZZI, Singularities in a modified Kuramoto-Sivashinsky equation describing interface motion for phase transition, Physica D 85 (1995), 375-404.

[8] A. J. Bernoff, A. L. BerTOZZI \& T. P. WiTElski, private communication, 1999.

[9] A. L. BERTOZZI, The mathematics of moving contact lines in thin liquid films, Notices Amer. Math. Soc. 45 (1998), 689-697.

[10] A. L. Bertozzi, M. P. Brenner, T. F. Dupont, \& L. P. Kadanoff, Singularities and similarities in interface flow, In: Trends and Perspectives in Applied Mathematics (L. Sirovich, ed.), Applied Mathematical Sciences, Volume 100, Springer-Verlag, New York, 1994, pp. 155208.

[11] A. L. BertozzI \& M. PUGH, The lubrication approximation for thin viscous films, the moving contact line with a 'porous media' cut off of Van der Waals interactions, Nonlinearity 7 (1994), 15351564 .

[12] The lubrication approximation for thin viscous flms, regularity and long time behavior of weak solutions, Commun Pur Appl Math 49 (1996), 85-123.

[13] _ Long-wave instabilities and saturation in thin film equations, Commun Pur Appl Math 51 (1998), 625-661.

[14] M. P. Brenner, P. Constantin, L. P. Kadanoff, A. Schenkel \& S. C. Venkataramani, Diffusion, attraction and collapse, Nonlinearity 12 (1999), 1071-1098.

[15] P. Constantin, Some mathematical problems of fluid mechanics, In: Proceedings of the International Congress of Mathematicians, Vol. 1, 2 (Zürich, 1994), Basel, Birkhäuser, 1995, pages 1086-1095.

[16] R. J. Deissler \& A. Oron, Stable localized patterns in thin liquid films, Phys Rev Lett 68 (1992), 2948-2951.

[17] T. F. Dupont, R. E. Goldstein, L. P. Kadanoff \& S.-M. Zhou, Finite-time singularity formation in Hele Shaw systems, Phys Rev E 47 (1993), 4182-4196.

[18] E. B. DUSSAN V, On the spreading of liquids on solid surfaces, static and dynamic contact angles, Ann Rev Fluid Mech 11 (1979), 371-400.

[19] A. Friedman, Partial Differential Equations, Holt, Rinehart, \& Winston, New York, 1969.

[20] D. Gilbarg \& N. S. Trudinger, Elliptic partial Differential Equations of Second Order, second edition, Springer-Verlag, Berlin-New York, 1983.

[21] R. T. GLASSEY, On the blowing up of solutions to the Cauchy problem for nonlinear Schrödinger equations, J. Math. Phys. 18 (1977), 1794-1797.

[22] R. Goldstein, A. Pesci \& M. Shelley, Instabilities and singularities in Hele-Shaw flow, Phys Fluids 10 (1998), 2701-2723.

[23] H. P. GREENSPAN, On the motion of a small viscous droplet that wets a surface, J Fluid Mech 84 (1978), 125-143.

[24] P. J. Haley \& M. J. Miksis, The effect of the contact line on droplet spreading, J Fluid Mech 223 (1991), 57-81.

[25] T. HOCHERMAN \& P. ROSENAU, On KS-type equations describing the evolution and rupture of a liquid interface, Physica D 67 (1993), 113-125.

[26] L. M. HockING, Rival contact-angle models and the spreading of drops, J Fluid Mech 239 (1992), 671-681.

[27] R. S. LAUgesen \& M. C. PUGH, Linear stability for steady states of thin film equations, Arch Ration Mech Anal 154 (2000), 3-51. 
[28] R. S. LAugesen \& M. C. PUgh, Properties of steady states for thin film equations, European J Appl Math 11 (2000), 293-351.

[29] H. A. LEVINE, Instability and nonexistence of global solutions to nonlinear wave equations of the form $P u_{t t}=-A u+\mathcal{F}(u)$, Trans. Amer. Math. Soc. 192 (1974), 1-21.

[30] A. MAJDA, Vorticity and the mathematical theory of incompressible fluid flow, Commun Pur Appl Math, 39(S, suppl.), S187-S220, 1986. Frontiers of the mathematical sciences, 1985 (New York, 1985).

[31] T. G. Myers, Thin films with high surface tension, SIAM Rev. 40(3) (1998) (electronic), 441462.

[32] A. Novick-Cohen, A singular minimization problem for droplet profiles, Eur J Appl Math 4 (1993), 399-418.

[33] A. Oron, S. H. Davis \& S. G. BanKOFF, Long-scale evolution of thin liquid films, Rev Mod Phys 69(3) (1997), 931-980.

[34] A. ORON \& P. RosenaU, Formation of patterns induced by thermocapillarity and gravity, J Phys II 2 (1992), 131-146.

[35] F. ОТTО, Lubrication approximation with prescribed non-zero contact angle, an existence result, Commun Part Diff Eq 23 (1998), 2077-2164.

[36] R. DAL PASSO \& H. GARCKE, Solutions of a fourth order degenerate parabolic equation with weak initial trace, Ann. Scuola Norm. Sup. Pisa Cl. Sci. (4) 28 (1999), 153-181.

[37] R. DAL PASSO, H. GARCKE \& G. GRÜN, On a fourth order degenerate parabolic equation, Global entropy estimates and qualitative behavior of solutions, SIAM J Math Anal 29 (1998), 321342.

[38] L. A. Peletier, The porous media equation, In, Applications of Nonlinear Analysis in the Physical Sciences, (H. Amman \& al., eds.), Pitman, New York, 1981, pp. 229-241.

[39] Alexander A. Samarskit, Victor A. Galaktionov, Sergei P. Kurdyumov \& AleXander P. Mikhailov, Blow-up in Quasilinear Parabolic Equations, Walter de Gruyter $\&$ Co., Berlin, 1995. Translated from the 1987 Russian original by Michael Grinfeld and revised by the authors.

A. L. BERTOZZI:

Departments of Mathematics and Physics, and

the Center for Nonlinear and Complex Systems

Duke University

Durham, NC 27708, U.S.A.

E-MAIL: bertozzi@math.duke.edu

M. C. Pugh:

Department of Mathematics

University of Pennsylvania

Philadelphia, PA 19104, U.S.A.

E-MAIL: mpugh@math.upenn.edu

1991 Mathematics Subject Classification: Primary number: 35K65; secondary: 35K30, 35B33, 76T10.

Received: October 26th, 1999; revised: March 10th, 2000. 